\title{
Automated Dating of the World's Language Families Based on Lexical Similarity
}

\author{
by Eric W. Holman, Cecil H. Brown, Søren Wichmann, André Müller, \\ Viveka Velupillai, Harald Hammarström, Sebastian Sauppe, \\ Hagen Jung, Dik Bakker, Pamela Brown, Oleg Belyaev, \\ Matthias Urban, Robert Mailhammer, Johann-Mattis \\ List, and Dmitry Egorov
}

CA+ Online-Only Material: Supplement A PDF

\begin{abstract}
This paper describes a computerized alternative to glottochronology for estimating elapsed time since parent languages diverged into daughter languages. The method, developed by the Automated Similarity Judgment Program (ASJP) consortium, is different from glottochronology in four major respects: (1) it is automated and thus is more objective, (2) it applies a uniform analytical approach to a single database of worldwide languages, (3) it is based on lexical similarity as determined from Levenshtein (edit) distances rather than on cognate percentages, and (4) it provides a formula for date calculation that mathematically recognizes the lexical heterogeneity of individual languages, including parent languages just before their breakup into daughter languages. Automated judgments of lexical similarity for groups of related languages are calibrated with historical, epigraphic, and archaeological divergence dates for 52 language groups. The discrepancies between estimated and calibration dates are found to be on average $29 \%$ as large as the estimated dates themselves, a figure that does not differ significantly among language families. As a resource for further research that may require dates of known level of accuracy, we offer a list of ASJP time depths for nearly all the world's recognized language families and for many subfamilies.
\end{abstract}

The greater the degree of linguistic differentiation within a stock, the greater is the period of time that must be assumed for the development of such differentiations.

(Sapir 1916:76)

Glottochronology, as formulated by Morris Swadesh (1950, 1955), is a method for estimating the amount of elapsed time since phylogenetically related languages diverged from a common ancestral language. This approach involves determining the percentage of words that are cognate in a standard list of basic vocabulary. Working with the assumption that words

Eric W. Holman is Professor Emeritus in the Department of Psychology at the University of California, Los Angeles (405 Hilgard Avenue, Los Angeles, California 90095-1563, U.S.A.). Cecil H. Brown is Distinguished Research Professor Emeritus at Northern Illinois University and Faculty Associate at the University of West Florida (1700 Scenic Highway, Apartment 601, Pensacola, Florida 32503, U.S.A. [brown.cecil@yahoo.com]). Søren Wichmann is Senior for items on such a list are replaced in individual languages over time at a more or less constant rate, Swadesh devised a formula, using cognate percentage as input, for calculating the length of time since a language divergence occurred.

Scientist in the Department of Linguistics at the Max Planck Institute for Evolutionary Anthropology (Deutscher Platz 6, D-04103 Leipzig, Germany). André Müller is a student of linguistics and Chinese studies at the University of Leipzig (Fachschaftsrat Linguistik, Geisteswissenschaftliches Zentrum, Beethovenstraße 15, D-04107 Leipzig, Germany). Viveka Velupillai is Research Fellow in the Department of English at the Justus-Liebig-Universität Gießen (OttoBehaghel-Straße 10 B, D-35394 Gießen, Germany). Harald Hammarström is Postdoctoral Fellow in the Centre for Language Studies at Radboud Universiteit (Postbus 9103, NL-6500 HD Nijmegen, The Netherlands) and in the Department of Linguistics at the Max Planck Institute for Evolutionary Anthropology (Deutscher Platz 6, D-04103 Leipzig, Germany). Sebastian Sauppe is Research Assistant in the Department of Linguistics at the Max Planck Institute for Evolutionary Anthropology (Deutscher Platz 6, D-04103 Leipzig, Germany). Hagen Jung is Computer Scientist at 
Glottochronology has had a checkered history since its formulation some 60 years ago. An early review by Hymes (1960) was generally favorable. Later, Embleton (1986) provided a judicious summary of both positive and negative views. More recently, the pros and cons of the method were discussed in numerous chapters of a collection edited by Renfrew, McMahon, and Trask (2000). We do not intend to continue the debate on the theoretical merits and demerits of glottochronology. Instead, we describe a new approach that infers language divergence from lexical similarity without the protracted linguistic analysis required for cognate identification.

Several distinct processes can cause lexical similarity among genetically related languages to diminish with the passage of time. One process is systematic change in sounds, whereby commonly inherited words in related languages become phonologically different. Other processes involve replacement of words by totally different words for the same referents. Such replacement may be due to conditions internal to individual languages, such as processes of semantic change or the borrowing of a word from one language into another where it is then used as a substitute for a native word. If two languages copy the same word from a third source, then borrowing may actually increase the similarity between the two languages. For lexical similarity to be useful for dating, the net effect of all these processes must be to reduce similarity at an approximately constant rate through time.

This paper describes a large-scale empirical test of the accuracy of dates produced when assuming a constant rate of decrease for lexical similarity. The test is performed on a database of computer-readable basic vocabulary lists for about one-half of the world's recorded languages. Judgment of lexical similarity is entirely automated and therefore approaches total objectivity. For a set of 52 language groups, lexical similarity determined through automation is calibrated with his-

the Max Planck Institute for Evolutionary Anthropology (Deutscher Platz 6, D-04103 Leipzig, Germany). Dik Bakker is Associate Professor at the University of Amsterdam and Visiting Scholar at the Linguistics Department of Lancaster University (Lancaster LA3 4YT, United Kingdom). Pamela Brown is an independent scholar (1700 Scenic Highway, Apartment 601, Pensacola, Florida 32503, U.S.A.). Oleg Belyaev is Junior Researcher in the Department of Typology at the Institute of Linguistics of the Russian Academy of Sciences, Moscow (1/1 Bolshoy Kislovsky Lane, Room 50, Moscow 125009, Russia). Matthias Urban is a $\mathrm{PhD}$ student in the Department of Linguistics at the Max Planck Institute for Evolutionary Anthropology (Deutscher Platz 6, D-04103 Leipzig, Germany). Robert Mailhammer is Assistant Professor in the Department of English at Arizona State University (P.O. Box 870302, Tempe, Arizona 85287-0302, U.S.A.). Johann-Mattis List is Research Assistant at the Institute of Romance Languages and Literature at Heinrich Heine University (Universitätsstraße 1, Building 23.21, Room 00.44A, 40225 Düsseldorf, Germany). Dmitry Egorov is Junior Research Fellow in the Education and Research Center for Linguistics at Kazan Federal University (18 Kremlyovskaya Street, Kazan 420008, Russia). This paper was submitted 11 XII 09 and accepted 12 I 11. torical, epigraphic, and archaeological dates of language divergence gathered from published sources. This calibration not only facilitates estimation of dates but also allows quantitative evaluation of the accuracy of the calculated dates. The observed level of accuracy can serve as the basis for informed decisions as to how to use dates calculated by the same method for other groups.

\section{The Automated Similarity Judgment Program Project}

The present approach is developed within the Automated Similarity Judgment Program (ASJP), ${ }^{1}$ first described by Brown et al. (2008). Brown et al. also review previous research on computerized lexicostatistics, which commenced with Grimes and Agard (1959). A major goal of ASJP is the development of a database of Swadesh (1955) lists for all of the world's languages, with all words transcribed into a standard orthography called “ASJPcode.” Brown et al. (2008:306-307) give a description of this orthography, including International Phonetic Alphabet equivalents of the ASJPcode symbols. The principal advantage of ASJPcode is that it can be produced with any QWERTY keyboard and thus is highly accessible to transcribers; a disadvantage is that it ignores some features such as tone, vowel length, and suprasegmental traits. A computer program was written to measure the overall lexical similarity of all possible pairs of languages in the database. In Brown et al. (2008) the program was applied to a database consisting of 100-item Swadesh lists from 245 globally distributed languages, all transcribed into ASJPcode. The automated lexicostatistical classifications of many language families were found to be similar to classifications by expert historical linguists.

Holman et al. (2008) subsequently determined the relative stability of each item on the 100-referent list. A subset of the 40 most stable of the 100 items was found to yield lexicostatistical results (in terms of their correlation with language classifications by specialists) at least as accurate as those produced by the full 100-item list. The shorter list facilitated a substantial increase in rate of language list production, as did the addition to the project of new transcribers. As a result, the database now (March 2011) consists of lists for 4,817 languages and dialects. The worldwide distribution of these is shown on the map in figure 1. Because some lists are for

1. Consult http://email.eva.mpg.de/ wichmann/ASJPHomePage.htm for full details on ASJP, including references to sources of data. Especially rich sources are the Austronesian Basic Vocabulary Database (http:// language.psy.auckland.ac.nz/austronesian/) described by Greenhill, Blust, and Gray (2008); the African data of Kropp-Dakubu (1977-1980); the Rosetta Project (http://www.rosettaproject.org); and the now defunct online database for South American languages maintained by the late Lincoln Ribeiro (formerly posted as http://paginas.terra.com.br/educacao/ GICLI/ListasEnglish.htm). We are particularly grateful to the more than 70 scholars who have contributed original field data. 


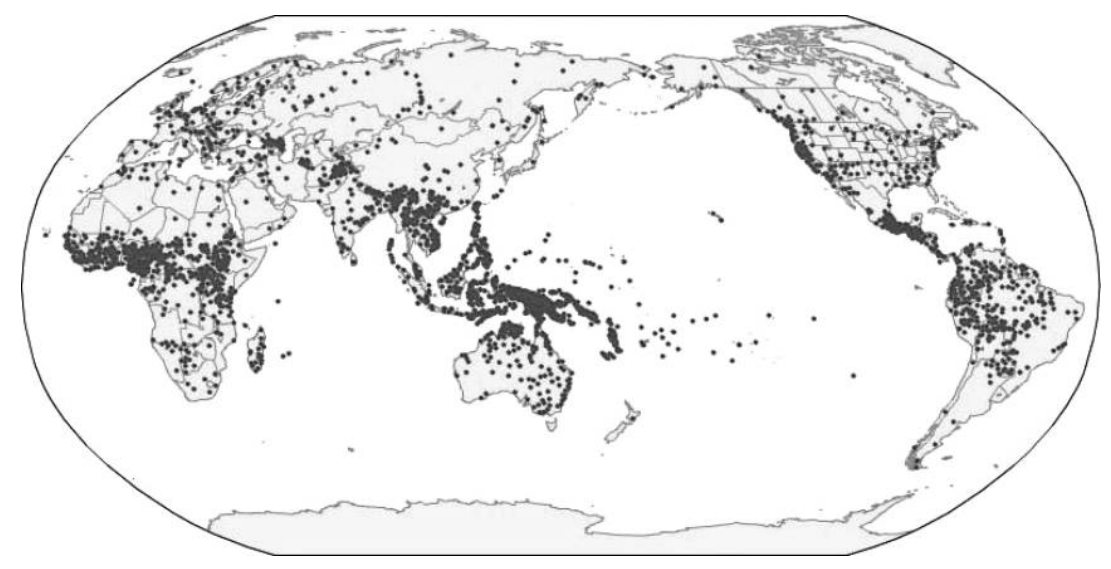

Figure 1. Distribution of languages and dialects in the Automated Similarity Judgment Program database. A color version of this figure is available in the online edition of Current Anthropology.

dialects of the same language, the set of lists represents 3,389 of the 6,779 spoken languages with different ISO639-3 designations in the sixteenth edition of Ethnologue (Lewis 2009), the most recent worldwide catalog of languages.

ASJP now employs a different similarity judgment program that produces even better lexicostatistical results compared with language classification by specialists. This program is based on Levenshtein distance (LD), also known as edit distance. Beginning with Kessler (1995), LD has previously been applied to language dialects. Kessler (1995) also reviewed earlier quantitative comparisons of dialects going back to Séguy (1971). To our knowledge, Serva and Petroni (2008) were the first to use LD to calculate language-group dates.

$\mathrm{LD}$ is defined as the minimum number of successive changes necessary to convert one word into another, where each change is the insertion, deletion, or substitution of a symbol. For example, in ASJPcode the Spanish word for "bone" is weso and the Italian word is osso. In order to convert the Spanish transcription to the Italian one, one insertion, one deletion, and one substitution are required: $s$ is added to the Spanish word, $w$ is deleted, and $o$ is substituted for $e$. Alternatively, to convert the Italian transcription to the Spanish one, $w$ is added word-initially to the Italian form, $s$ is deleted, and $e$ is substituted for the first $o$. Either way, the Spanish and Italian words demonstrate an LD of 3. This symmetry holds in general because deletions and insertions are the inverse of one another and substitutions are not sensitive to the direction of change. Paired words with smaller LDs are more lexically similar than those with larger LDs.

Levenshtein measurement of similarity treats all changes as equivalent without regard to their phonological plausibility or historical frequency. In comparisons between dialects, Kessler (1995) and Heeringa et al. (2006) explored generalizations of LD in which some changes contribute more to LD than others. These generalizations did not improve the cor- relations of LD with any of several external criteria. Consistent with these findings, early attempts within the ASJP project to incorporate phonological information into automated similarity judgment did not augment correlations with classifications by specialists and in some instances even lowered them.

Within the Levenshtein approach, differences in word length can be corrected for by dividing LD by the number of symbols of the longer of the two compared words. This produces normalized LD (LDN), which was used by Serva and Petroni (2008). ASJP includes synonyms on its lists but no more than two per meaning. For referents represented by two synonyms, LDN is the average LDN of the two. For a given pair of languages, LDN for paired words having the same meaning in the two languages is averaged across all the meanings on the list attested by words in both languages. As a baseline for phonological distance independent of meaning, LDN is also averaged across all pairs with different meanings attested in the two languages. An LDN divided (LDND) between the two languages is calculated by dividing the average LDN for all the word pairs involving the same meaning by the average LDN for all the word pairs involving different meanings. ${ }^{2}$ As a result, the distance measured by LDND is specifically lexical rather than phonological (Wichmann et al. $2010 a$ ). Finally, to produce a measure of lexical similarity analogous to the cognate percentages used by Swadesh and others, ASJP similarity (abbreviated $s$ ) is defined as $1-$ LDND. Similarity is $100 \%$ by definition between identical lists without synonyms, and similarity is near $0 \%$ on average be-

2. More formally, let two languages, A and B, be given, and let $n$ be the number of items (out of 40) attested in both languages. Let $d_{i j}$ denote LDN between item $i$ in language $\mathrm{A}$ and item $j$ in language $\mathrm{B}$. Then $\mathrm{LDND}=\left[\sum_{i}\left(d_{i i}\right) / n\right] /\left[\sum_{i \neq j}\left(d_{i j}\right) / n(n-1)\right]$. 
tween lists from languages that are not at all related by either descent or contact.

Automated use of LD to measure lexical similarity of languages eliminates human judgment of similarity and the ambiguities this entails. Over the years, glottochronology has produced dates for many groups rendered by many different linguists using their individual approaches to cognate identification. In well-studied language families, cognates can be determined rigorously, but this procedure is very labor intensive and has not been achieved for most families. This lack of uniformity was considered a serious problem in a very early discussion of glottochronology by Swadesh (1955:129; cf. Hymes 1960:18-19). ASJP chronology, in contrast, provides a uniform method for judging lexical similarity and for dating all of the world's phylogenetic language groups, including those for which cognate matches have yet to be worked out.

\section{Lexical Heterogeneity at Time Zero}

Swadesh (1950) proposed that if $t$ is the time since two related languages diverged from each other and $C$ is the proportion of items on a basic vocabulary list that are cognate between the two languages, then $t$ can be estimated based on the hypothesis that, on average,

$$
t=\frac{\log C}{2 \log r},
$$

where $r$ is the average proportion of items on the list that are retained after a standard time period (usually 1,000 years). This formula can be modified for ASJP chronology by replacing the cognate proportion $C$ with the ASJP similarity $s$, which has been defined as 1 - LDND:

$$
t=\frac{\log s}{2 \log r} .
$$

In other words, $s$, which is a similarity score derived from an LD, can be used exactly like a proportion of shared cognates in glottochronology to estimate time depth. In formula (2), $r$ is the average proportion of lexical similarity retained after a standard period of time.

Both formulas (1) and (2) assume that genetically related languages diverged from a single ancestral language that was spoken at $t=0$, or time zero, the point immediately before the ancestral language began to split into daughter languages. Substitution of $t=0$ into formula (1) implies that $\log C=$ 0 , which in turn implies that $C=1$, which corresponds to $100 \%$ lexical homogeneity for speakers of a single language at time zero. A similar substitution of $t=0$ into formula (2) also implies $100 \%$ lexical homogeneity at time zero. This is an oversimplification, as Hymes (1960:26-27) recognized. If a time-zero language comprised a chain or network of dialects (Ross 1988:8), then lexical variation almost certainly existed across them. Even if no dialectal diversity were apparent, it is unlikely that any time-zero languages were ever totally lexically homogeneous, because all languages tend to show some variation across speakers even if distinct dialects are not observed. Consequently, formula (2) should be revised to capture formally the heterogeneity of time-zero languages. For this purpose, we let $s_{0}$ represent the average degree of lexical similarity within time-zero ancestral languages. Therefore, the quantity that should start at 1 when $t=0$ in formula (2) is not $s$ itself but rather the ratio $s / s_{0}$. Because $\log s / s_{0}=$ $\log s-\log s_{0}$, the revised version of formula (2) is

$$
t=\frac{\log s-\log s_{0}}{2 \log r} .
$$

\section{Calibration Procedure}

Once values are established for $s, s_{0}$, and $r$ in formula (3), they can be used in the equation to yield a solution for $t$, the time depth of a language divergence. The value of $s$ is determined from the data through Levenshtein analysis. The values of $s_{0}$, the average degree of lexical similarity within time-zero languages, and of $r$, the average proportion of lexical similarity retained after a standard period of time, are constants in the formula. Thus, in order to solve for $t$, the constants $s_{0}$ and $r$ must be known. The standard empirical method for determining $s_{0}$ and $r$ is linear regression.

Linear regression requires a set of calibration points for groups of genetically related languages with known $t$ and $s$. The value of $t$ is the date at which the group's ancestral language first began to break apart as determined from published epigraphic, historical, or archaeological sources. For the few languages with written materials dated near the time of their divergence, the dates can be determined more or less directly. Otherwise, if speakers of the languages have a recorded history, dates of divergence can be inferred indirectly from the dates of events expected to impair communication between communities, such as migration to places distant from each other or long-term domination by mutually antagonistic states. For dates before recorded history, archaeology can be used for calibration if words for archaeologically datable objects can be traced to ancestral languages or if a currently observable association between a language group and a characteristic type of material culture can be extrapolated into the archaeological past. In addition, some of the calibration sources establish dates by correlating loanwords with historically or archaeologically datable periods of contact between languages. As Heggarty (2007) has cogently argued, there are various difficulties in identifying languages with archaeological materials. We nevertheless use archaeological calibrations because they are the only ones available for chronologically deep families.

In addition to the criteria for including calibration points, we also invoke a criterion for excluding candidates. Some sources infer dates for language divergence from archaeological or historical information combined with glottochronol- 
ogy or estimates of similarity between languages. We exclude all potential calibration points of this sort. Our combination of criteria for inclusion and exclusion is intended to identify the most reliable dates that investigators have so far been able to glean from information that is independent of linguistic similarity.

In total, we have assembled 52 published calibration dates that satisfy the criteria described above and for which the ASJP database contains the relevant languages, including those that became extinct after $1900 \mathrm{CE}$ as well as those currently spoken. By analogy with glottochronology, the similarity score $s$ for a language family or subfamily is based on pairwise similarities between the extant (or recently extinct) languages in its highest-level coordinate subgroups. For instance, IndoIranian is divided at the highest level into Indic and Iranian, and its similarity score is estimated from the similarities of pairs, each consisting of an Indic and an Iranian language.

The similarities between such subgroups can be used in different ways to estimate overall similarity for a family or subfamily. One possibility, analogous to the suggestion by Swadesh (1950) for glottochronology, is to use only the smallest similarity score among all the language pairs compared, because the observed lexical similarity for the two least similar languages is least likely to have been influenced by diffusion of words between languages in different subgroups. Other possibilities are the median or the mean of the similarities. ASJP uses the mean for the following reasons. First, Holman et al. (2008, fig. 3) found that for similarities based on the 40-item list, diffusion has much less effect than phylogenetic relationship. Second, at least one of the pairs of languages with minimum similarity may be a geographic outlier that is atypically different from its sister languages because of removal from them and contact with other languages. Third, the sampling distribution of the mean is less variable than the sampling distributions of the minimum or the median.

The mean similarity calculated for a genetic group such as a language family is directly influenced by the way in which its member languages are sorted into subgroups. In the literature, different classifications are often reported for the same language family, each showing a different set of highestlevel coordinate subgroups. Which of these classifications is closest to phylogenetic reality is not always obvious, even to those who have specialized knowledge of a specific language family. To minimize the effect of this ambiguity, we require each of our calibration points to be compatible not only with the classification (if any) provided in the source of the calibration date but also with the classification in the sixteenth edition of Ethnologue (Lewis 2009). For the most part, Ethnologue appears to be based on previously published classifications, although the sources of the classifications are not cited.

The classifications in Ethnologue of some families are more conservative than those in the calibration sources. For instance, the calibration sources for the Turkic languages distinguish Chuvash from the other surviving Turkic languages, collectively called Common Turkic. Ethnologue does not make this distinction, instead listing Chuvash among six coordinate subgroups at the highest level within Turkic. Nevertheless, Common Turkic can be constructed by combining the five subgroups other than Chuvash. As a general definition, groups from calibration sources are compatible with Ethnologue if and only if they can be formed by combining coordinate Ethnologue subgroups without moving any languages from one subgroup to another. If a date in a calibration source refers to a group that is not compatible with the Ethnologue classification in this sense, we do not use that date.

Classifications from calibration sources and Ethnologue are the only guides for language subgroups used for calibration in this study. Through this restrictive approach, ASJP avoids the subjectivity entailed in making choices between competing classifications that could be biased toward a particular result.

\section{Calibration Points}

In the following list, the 52 language groups are presented in alphabetical order. A calibration date in years BP is given for each group, followed by an abbreviation indicating whether the date is based on epigraphic $(\mathrm{E})$, historical $(\mathrm{H})$, or archaeological (A) information. The published information itself is described in an immediately following "source" section. When the source gives a range of dates, the middle of the range is used; for convenience, the present time is taken to be the year 2000. Next, the average similarity score is given for the group. This is based on the subgroups listed subsequently, which are named as in Ethnologue unless otherwise indicated (each subgroup is followed in parentheses by the number of ASJP lists it contains). The last figure is the number of pairwise language comparisons averaged to produce the score.

\section{Benue-Congo}

Date: 6500 (A).

Source: Bostoen and Grégoire (2007:77) link the introduction, during 7000-6000 BP, of new technologies such as macrolithic tools and pottery into the Grassfields region with the break off of Bantoid from Benue-Congo. They argue that this would fit the hypothesis that the center of dispersion of Benue-Congo is near the confluence of the Niger and Benue rivers, and they also mention that pottery-related terminology can be reconstructed to Proto-Benue-Congo.

Similarity: 3.58 .

Comparisons: Akpes (1), Bantoid (258), Cross River (28), Defoid (4), Edoid (27), Idomoid (3), Igboid (5), Jukunoid (2), Kainji (20), Nupoid (4), Oko (1), Plateau (45), Ukaan (6); 46,303 pairs.

\section{Brythonic}

Date: $1450(\mathrm{H})$.

Source: Humphreys (1993:609) concludes from the avail- 
able historical and linguistic evidence that the distinctiveness of Breton stems from British immigration mainly from the fifth to the seventh centuries CE.

Similarity: 42.60 .

Comparisons: Breton (1), Welsh (1); 1 pair.

\section{Central Southern African Khoisan}

Date: 2000 (A).

Source: Güldemann (forthcoming:16) associates the ancestors of the Central Southern African Khoisan (or Khoe-Kwadi, in his terminology) with a cultural sequence starting around 2000 BP, which marks the introduction of food production in the part of Africa where Central Southern African Khoisan speakers are currently located. Support for this hypothesis is provided by the word * $g u$, "sheep," which, according to the author, can be reconstructed for the entire language group and which has been borrowed widely into Bantu.

Similarity: 11.67.

Comparisons: Nama-Tshu-Khwe (6), Kwadi (1); 6 pairs.

Note: Nama-Tshu-Khwe subgroup is from Güldemann (forthcoming).

\section{Cham}

Date: $529(\mathrm{H})$.

Source: According to Thurgood (1999:44), "there is really no question about the relationship between Western and Phan Rang Cham, as they were the same language until the fall of the southern capital in 1471."

Similarity: 55.06.

Comparisons: Eastern Cham (1), Western Cham (1); 1 pair.

\section{Chamic}

Date: $1550(\mathrm{H})$.

Source: Sidwell (2006:198-199) suggests that the breakup of Chamic followed a migration of Chams to Aceh under pressure from Chinese attacks during the fifth century CE.

Similarity: 15.90.

Comparisons: Acehnese (1), Coastal-Highlands Chamic (6); 6 pairs.

Note: Coastal-Highlands Chamic subgroup is from Sidwell (2006).

\section{Chinese}

Date: $2000(\mathrm{H})$.

Source: According to Norman (1988:185), the imperial expansion under the Qin and Han dynasties first brought the Chinese language to what are today the Guangdong, Guangxi, Fujian, and southern Jiangxi provinces. This colonization, he argues, was the origin of the differences among modern Chinese languages, particularly the southern dialect group.

Similarity: 12.97.
Comparisons: Hakka (1), Mandarin (2), Min Nan (2), Wu (1), Yue (1); 19 pairs.

Cholan

Date: 1600 (E).

Source: Wichmann (2006:283) argues on the basis of epigraphic evidence that Eastern and Western Cholan had split into dialects by $400 \mathrm{CE}$.

Similarity: 43.26 .

Comparisons: Chorti (2), Chol-Chontal (3); 6 pairs.

Common Turkic (Turkic Languages minus Chuvash)

Date: $1419(\mathrm{H})$.

Source: In the account of Golden (1998:19-20), "Turkic now became the predominant linguistic element in Mongolia and the steppelands in and around what is now Turkestan and extending into the Pontic zone" in $552 \mathrm{CE}$, when Bumïn established the Türk Kaghanate. This empire, however, ceased to be united by the end of the rule of Taspar in $581 \mathrm{CE}$.

Similarity: 37.94.

Comparisons: Eastern (2), Northern (7), Southern (30), Western (11); 713 pairs.

Note: Common Turkic subgroup is from Golden (1998).

\section{Czech-Slovak}

Date: 1050 (E).

Source: Fodor (1962:132) states that "the linguistic unity of the Czech and Slovak languages dissolved in the 10th century."

Similarity: 67.18.

Comparisons: Czech (1), Slovak (1); 1 pair.

Dardic

Date: 3550 (A).

Source: Parpola (1999:200) correlates the Early Gandhara Grave culture (Ghalegay IV) in Swat (1700-1400 BCE) with Proto-Rgvedic, which he equates with Proto-Dardic.

Similarity: 26.97.

Comparisons: Chitral (9), Kashmiri (1), Kohistani (4), Kunar (3), Shina (5); 176 pairs.

\section{Eastern Malayo-Polynesian}

Date: 3350 (A).

Source: According to Pawley (2009:517), there is a strong association between the first appearance of nucleated villages in the Bismarck Archipelago in 3400-3300 BP and the arrival of Austronesian languages, "specifically with the separation of the large Oceanic branch from its nearest relatives, spoken in the Cenderawasih Bay area at the western end of New Guinea, and in South Halmahera." Similarity: 7.56 . 
Comparisons: Oceanic (428), South Halmahera-West New Guinea (43); 18,404 pairs.

\section{East Polynesian}

Date: 1050 (A).

Source: According to Bellwood and Hiscock (2005:290), radiocarbon dates indicate that the Marquesas, Societies, Cooks, Australs, Tuamotus, Hawaiian Islands, Easter Island, and New Zealand were settled starting around $700 \mathrm{CE}$ and ending several centuries later. Bellwood and Hiscock (2005: 292) report general acceptance of the interval between about 700 and $1200 \mathrm{CE}$ as the time when most of central and eastern Polynesia was colonized.

Similarity: 48.34.

Comparisons: Rapanui (1), Central (10); 10 pairs.

\section{East Slavic}

Date: $760(\mathrm{H})$.

Source: According to Pugh (2007:10-11), the sacking of Kiev by the Tatars in 1240 CE was soon followed by political fragmentation and linguistic divergence.

Similarity: 39.47.

Comparisons: Belarusan (1), Russian (2), Ukrainian (1); 5 pairs.

\section{English-Frisian}

Date: $1550(\mathrm{H})$.

Source: Bremmer (2009:125) states that the Anglo-Saxon conquest of Britain in the fifth century CE implied a separation of what would later become English from the immediate ancestor of Frisian.

Similarity: 30.57.

Comparisons: English (2), Frisian (2); 4 pairs.

Note: English-Frisian subgroup is from Bremmer (2009).

\section{Ethiopian Semitic}

Date: 2450 (E).

Source: According to Ehret (2000:387), the ancestral language of all the members of Ethiopian Semitic is attested in epigraphic records dating to the fifth century BCE at sites in modern-day Eritrea and northern Ethiopia. The relatively wide distribution of epigraphic evidence for the language suggests a geographic dispersal of its speakers and thus the beginning of its breakup.

Similarity: 18.90.

Comparisons: North (4), South (14); 56 pairs.

\section{Ga-Dangme}

Date: 600 (A, H).

Source: Ehret (2000:390-391) states that "the protoDangme people of southern Ghana can be tied through both oral tradition and material culture traits to a particular development of town life along the lower Volta River, belonging in the archaeology to the period 1200-1400. Beginning in the fifteenth century, this culture diverged into a set of independent polities, most often consisting of a town and its immediately surrounding rural area."

Similarity: 49.11.

Comparisons: Dangme (1), Ga (1); 1 pair.

\section{Germanic}

Date: $2100(\mathrm{H})$.

Source: The emergence of the Cimbri and the Teutones toward the end of the second century BCE (Pohl 2004:11) was the beginning of the migrations associated with the breakup of Germanic.

Similarity: 29.24.

Comparisons: North (7), West (23); 161 pairs.

\section{Goidelic}

Date: 1050 (E).

Source: According to Jackson (1951:91-92), the Gaelic of Ireland, Scotland, and the Isle of Man was identical up until the tenth century $\mathrm{CE}$, but from that century onward there are indications of divergence between Eastern and Western Gaelic.

Similarity: 27.52.

Comparisons: Irish Gaelic (1), Scottish Gaelic-Manx (2); 2 pairs.

Note: Scottish Gaelic-Manx subgroup is from Jackson (1951).

\section{Hmong-Mien}

Date: 2500 (E).

Source: Sagart, Blench, and Sanchez-Mazas (2005:2-3) date Proto-Hmong-Mien to 2500 BP based on the phonological shapes and cultural contents of early loanwords. More specifically, Sagart (1999:208) discusses the Chinese word for "money," which is among the borrowings into Hmong-Mien. According to Sagart (Laurent Sagart, e-mail, August 4, 2010), this word is first attested in Chinese texts in the fifth century BCE.

Similarity: 5.66.

Comparisons: Hmongic (9), Ho Nte (1), Mienic (4); 49 pairs.

Indo-Aryan (Indic)

Date: 3900 (A).

Source: Parpola (1999:200) correlates Early Andronovo (Petrovka; ca. 2000-1800 BCE) with Proto-Indo-Aryan.

Similarity: 24.79 .

Comparisons: Central group (1), Central zone (44), Eastern zone (3), Northern zone (1), Northwestern zone (39), Nu- 
ristani (2), Sinhalese-Maldivian (2), Southern zone (1); 2,586 pairs.

Indo-European (Minus Anatolian and Tocharian)

Date: 5500 (A).

Source: Anthony (1995:558) argues that Proto-IndoEuropean "existed as a single speech community late enough to experience and create words for wheeled vehicles" and that it cannot have differentiated until after 3500 BCE. Nichols and Warnow (2008:781) then give $5500 \mathrm{BP}$ as a benchmark date for the breakup of Indo-European but mention a slightly earlier divergence of Anatolian, which is not included in this calibration.

Similarity: 5.29 .

Comparisons: Albanian (1), Armenian (2), Baltic (2), Celtic (5), Germanic (30), Greek (1), Indo-Iranian (147), Romance (14), Slavic (16); 12,264 pairs.

\section{Indo-Iranian}

Date: 4400 (A).

Source: Parpola (1999:200) correlates Proto-Aryan with the Catacomb Grave and Poltavka cultures (ca. 2800-2000 BCE). Similarity: 8.28 .

Comparisons: Indic (93), Iranian (54), 5,022 pairs.

Inuit

Date: 800 (A).

Source: Figure $2 b$ of Fortescue (1998:27) depicts NeoEskimo migration routes with dates and indicates a major dispersal starting around $1200 \mathrm{CE}$. According to Fortescue (1998:33), this date corresponds to "the first phase of the Thule entry into Greenland" and is based on (recalibrated) carbon 14 dates.

Similarity: 60.40 .

Comparisons: North Alaskan Inupiatun (1), Western Canadian Inuktitut (1), Eastern Canadian Inuktitut (1), Greenlandic Inuktitut (1); 6 pairs.

Iranian

Date: 3900 (A).

Source: Parpola (1999:200) correlates Proto-West-Aryan with the Early Timber Grave and Abashevo cultures (ca. 20001800 BCE).

Similarity: 14.09 .

Comparisons: Eastern (47), Western (7); 329 pairs.

\section{Italo-Western Romance}

Date: $1524(\mathrm{H})$.

Source: Although Bury (1923:408) argues against a common view that the revolution of $476 \mathrm{CE}$ implied the "fall of the Western Roman Empire," he does see it as marking the point at which the disintegration of the empire first extended to Italy.

Similarity: 32.15 .

Comparisons: Italo-Dalmatian (2), Western (10); 20 pairs.

\section{Ket-Yugh}

Date: $1300(\mathrm{H})$.

Source: Vajda (forthcoming:5) claims that the divergence of Ket and Yugh dates to after the Kirghiz (Turkic) intrusion into the Yenisei region (ca. $700 \mathrm{CE}$ ).

Similarity: 48.65.

Comparisons: Ket (1), Yugh (1); 1 pair.

\section{Maa}

Date: $600(\mathrm{H})$.

Source: Ehret (2000:396) dates Proto-Maa to 600 BP. This is an approximate date based on oral traditions of the Maasai and some of their neighbors, which indicates that "the breakup of the Proto-Maa society and the emergence of a distinct Maasai society can be dated to not long before the sixteenth century" (Ehret 2000:385).

Similarity: 67.52 .

Comparisons: Maasai (1), Samburu (2); 2 pairs.

Note: Maa subgroup is from Ehret (2000).

\section{Ma'anyan-Malagasy}

Date: 1350 (A).

Source: Adelaar (2006:19) dates the migration of South East Barito speakers to Madagascar to the seventh century CE, after the foundation of Srivijaya.

Similarity: 30.30 .

Comparisons: Ma'anyan (2), Malagasy (18); 36 pairs.

Note: This subgroup is based on Dahl's (1951) identification of Ma'anyan as the language most similar to Malagasy, an identification restated by Ethnologue.

\section{Malayo-Chamic}

Date: 2400 (A).

Source: According to Sidwell (2006:199), Malayo-Chamic breaks up around 500-300 BCE, when Chamic speakers settle on the mainland and initiate contact with speakers of mainland languages.

Similarity: 24.67.

Comparisons: Malayic (23), Chamic (7); 161 pairs.

Note: Malayo-Chamic subgroup is from Sidwell (2006). Adelaar (2005) presents evidence that the Bali-Sasak-Sumbawa group diverged from Malayic and Chamic at about the same time in a three-way split, but to be conservative we use only Malayic and Chamic. 
Malayo-Polynesian

Date: 4250 (A).

Source: According to Bellwood (2007:40) "the first archaeological appearance to the south of Taiwan of Neolithic communities who used pottery and polished stone adzes, and kept pigs and dogs, occurred in the northern Philippines and western Borneo around 2500-2000 BC.”

Similarity: 12.62 .

Comparisons: Central-Eastern (580), Celebic (61), Chamorro (1), Enggano (3), Greater Barito (57), Javanese (3), Lampungic (24), Land Dayak (3), Malayo-Sumbawan (34), Moklen (1), North Borneo (17), Northwest Sumatra-Barrier Islands (4), Palauan (1), Philippine (151), Rejang (1), South Sulawesi (11); 268,972 pairs.

\section{Maltese-Maghreb Arabic}

Date: $910(\mathrm{H})$.

Source: According to Castillo (2006:29), Arabic domination of Malta lasted from 870 to 1090 CE.

Similarity: 33.57.

Comparisons: Maltese (1), Maghreb Arabic (3); 3 pairs.

\section{Mississippi Valley Siouan}

Date: 2475 (A).

Source: Rankin's (2006:574) table 41-3 shows Proto-Mississippi Valley Siouan breaking up between 2700 and 2250 BP. Rankin (2006:572) infers this date from the observation that the different Mississippi Valley Siouan languages have different words for squash, which became widely cultivated between 500 and 200 BCE.

Similarity: 28.23.

Comparisons: Chiwere (1), Dakota (3), Dhegiha (4), Winnebago (1); 27 pairs.

Mongolic

Date: $750(\mathrm{H})$.

Source: Janhunen (2003:3) describes the ancestral ProtoMongolic language as the result of intensive linguistic unification under the rule of Chinggiz Khan, and Weiers (2003: 248) states that "Moghol developed from the language spoken by the Mongols who during the thirteenth and fourteenth centuries were garrisoned in the west. . . As far as we know, the garrison Mongols who remained in the west never again had any contact with their kinsmen in Mongolia."

Similarity: 20.75.

Comparisons: Eastern (7), Western (1); 7 pairs.

\section{Northern Roglai-Tsat}

Date: $1000(\mathrm{H})$.

Source: According to Thurgood (1999:43), “Tsat and Northern Roglai represent a Northern Cham dialect that split into two under the impetus provided by the Vietnamese capture of the northern capital at Indrapura.... As late as around $1000 \mathrm{AD}$, these two languages probably constituted a single Northern Cham dialect."

Similarity: 25.83 .

Comparisons: Northern Roglai (1), Tsat (1); 1 pair.

Note: Northern Roglai-Tsat subgroup is from Thurgood (1999).

\section{Ongamo-Maa}

Date: 1150 (A).

Source: Referring to speakers of the Proto-Maa-Ongamo language, Ehret (2000:384-385) states, "Their arrival in central Kenya can be correlated with the appearance in the eighth century of a new pottery, Lanet ware, which has continued to be used by their descendants down to the present." Moreover, he claims that "the Maa-Ongamo separation took shape by or before $1000 \mathrm{AD}$, because the Proto-Chaga, a Bantu people of the period 1000-1200, were already by those centuries borrowing Maa-Ongamo words that showed the distinctive phonological features of Ongamo."

Similarity: 45.17.

Comparisons: Maa (3), Ngasa (1); 3 pairs.

Note: Maa subgroup is from Ehret (2000).

\section{Oromo}

Date: 460 (E).

Source: Ehret (2000:387), based on epigraphic evidence, dates the beginning of the expansion of Oromo people to 1530-1550 CE.

Similarity: 63.46.

Comparisons: Orma (1), Borana (2), Eastern (1), West Central (2); 13 pairs.

\section{Pama-Nyungan}

Date: 4500 (A).

Source: Evans and Jones (1997:417) link Proto-PamaNyungan to "new stone and food staple technologies and intensification in the archaeological record," including increased population density, new art styles, and the extension of long-distance trade networks. They argue that the family would have spread "something like 4000 to 5000 years ago" (Evans and Jones 1997:386).

Similarity: 5.47.

Comparisons: Arandic (5), Baagandji (1), Bandjalangic (2), Dyangadi (1), Dyirbalic (4), Galgadungic (2), Gumbaynggiric (2), Guugu-Yimidhirr (1), Iyora (1), Kala Lagaw Ya (1), Karnic (6), Kulinic (7), Maric (9), Muruwaric (1), Paman (21), South-West (23), Tangic (1), Waka-Kabic (4), Wiradhuric (3), Worimi (2), Yalandyic (1), Yanyuwan (1), Yidinic (2), Yotayotic (1), Yugambal (1), Yuin (3), Yuulngu (16); 6,693 pairs. 
Romance

Date: $1729(\mathrm{H})$.

Source: Watson (1999:155-156) gives $271 \mathrm{CE}$ as the most likely date for the withdrawal of the last Roman troops to the south of the Danube, after which the Latin language persisted north of the river to become Romanian.

Similarity: 28.96.

Comparisons: Eastern (2), Italo-Western (12); 24 pairs.

\section{Romani}

Date: $650(\mathrm{H})$.

Source: According to Matras (2002:1), references to "gypsies" in chronicles allow the reconstruction of "an outwards migration from the Balkans beginning in the fourteenth century, and reaching northern and western Europe in the fifteenth century."

Similarity: 61.92.

Comparisons: Balkan (7), Northern (12), Vlax (7), Dolenjski (1); 243 pairs.

Saami

Date: 1750 (A)

Source: Aikio (2006:43) dates the disintegration of ProtoSaami to approximately 0-500 CE mainly on the basis of the phonology and distribution of Proto-Scandinavian loanwords.

Similarity: 33.63.

Comparisons: Eastern (3), Western (3); 9 pairs.

\section{Scandinavian (North Germanic)}

Date: 1100 (E).

Source: Haugen (1982:9) states that by the time of the Viking period (ca. 750-1050 CE), a split is observable between East and West Scandinavian.

Similarity: 32.83 .

Comparisons: East (5), West (2); 10 pairs.

Slavic

Date: $1450(\mathrm{H})$.

Source: Schenker (1995:9, 15-17) quotes descriptions written in the sixth century CE of the geographic expansion and political anarchy of the Slavs, conditions that initiated the breakup of the common Slavic language.

Similarity: 43.01

Comparisons: East (4), South (6), West (6); 84 pairs.

Sorbian (Lusatian)

Date: 450 (E).

Source: Fodor (1962:132) states that "Lower and Upper Lusatian developed from the more or less homogeneous Lusatian in the 16th century, i.e., at the time of the reformation."
Similarity: 68.78.

Comparisons: Lower Sorbian (1), Upper Sorbian (1); 1 pair.

\section{Southern Nilotic}

Date: 2500 (A).

Source: Ehret (2000:385) correlates the Southern Nilotic languages with the Elmenteitan culture and then states that "in the sixth and fifth centuries BC, a major offshoot of the Elmenteitan moved into the vast Mara and Loita plains south of the western highlands," and he specifically correlates this offshoot with the Tatoga branch of Southern Nilotic.

Similarity: 13.43.

Comparisons: Kalenjin (5), Tatoga (6); 30 pairs.

\section{Southern Songhai}

Date: $550(\mathrm{H})$.

Source: According to Moraes Farias (2003:clxxiii), trade diasporas adopted Songhai as a lingua franca, probably during the expansion of the Songhai empire in the fifteenth century $\mathrm{CE}$, and then propagated the language farther to the south.

Similarity: 62.85 .

Comparisons: Dendi (1), Songhay (1), Koyra Chiini Songhay (1), Koyraboro Senni Songhay (1), Zarma (2); 14 pairs.

\section{Southwest Tungusic}

Date: $236(\mathrm{H})$.

Source: Ramsay (1987:216) identifies the Xibe as the descendents of Manchu who were resettled in Xinjiang as border guards in 1764.

Similarity: 53.27.

Comparisons: Xibe (1), Manchu (2); 2 pairs.

\section{Swahili}

Date: $1200(\mathrm{~A}, \mathrm{H})$.

Source: Ehret (2000:381) mentions both archaeological and

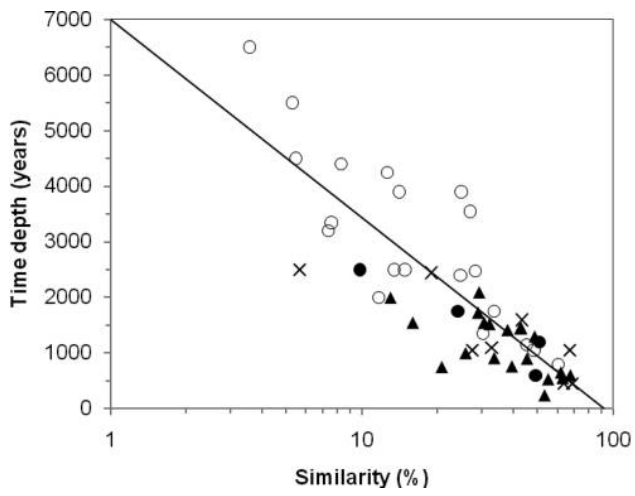

Figure 2. Time depth $(t)$ as a function of similarity $(s)$ with regression line; dates are archaeological (open circles), archaeological and historical (filled circles), historical (triangles), and epigraphic (crosses). 
Table 1. Comparison of calibration dates for 52 language groups with Automated Similarity Judgment Program (ASJP) dates based on $s_{0}=0.92$ and $r=0.72$

\begin{tabular}{|c|c|c|c|}
\hline Language group & Calibration date & ASJP date & Difference $(\%)$ \\
\hline \multicolumn{4}{|l|}{ Archaeological: } \\
\hline Benue-Congo & 6500 & 4940 & $-1,560(-32)$ \\
\hline Indo-European & 5500 & 4348 & $-1,152(-26)$ \\
\hline Pama-Nyungan & 4500 & 4295 & $-205(-5)$ \\
\hline Indo-Iranian & 4400 & 3665 & $-735(-20)$ \\
\hline Malayo-Polynesian & 4250 & 3024 & $-1,226(-41)$ \\
\hline Indo-Aryan (Indic) & 3900 & 1996 & $-1,904(-95)$ \\
\hline Iranian & 3900 & 2856 & $-1,044(-37)$ \\
\hline Dardic & 3550 & 1868 & $-1,682(-90)$ \\
\hline Eastern Malayo-Polynesian & 3350 & 3803 & $+453(+12)$ \\
\hline Temotu & 3200 & 3844 & $+644(+17)$ \\
\hline Southern Nilotic & 2500 & 2928 & $+428(+15)$ \\
\hline Wakashan & 2500 & 2781 & $+281(+10)$ \\
\hline Mississippi Valley Siouan & 2475 & 1798 & $-677(-38)$ \\
\hline Malayo-Chamic & 2400 & 2003 & $-397(-20)$ \\
\hline Central Southern African Khoisan & 2000 & 3143 & $+1,143(+36)$ \\
\hline Saami & 1750 & 1532 & $-218(-14)$ \\
\hline Ma'anyan-Malagasy & 1350 & 1690 & $+340(+20)$ \\
\hline Ongamo-Maa & 1150 & 1083 & $-67(-6)$ \\
\hline East Polynesian & 1050 & 979 & $-71(-7)$ \\
\hline Inuit & 800 & 640 & $-160(-25)$ \\
\hline \multicolumn{4}{|l|}{ Archaeological, historical: } \\
\hline Turkic & 2500 & 3404 & $+904(+27)$ \\
\hline Tupi-Guarani (coastal) & 1750 & 2043 & $+293(+14)$ \\
\hline Swahili & 1200 & 903 & $-297(-33)$ \\
\hline Ga-Dangme & 600 & 955 & $+355(+37)$ \\
\hline \multicolumn{4}{|l|}{ Historical: } \\
\hline Germanic & 2100 & 1745 & $-355(-20)$ \\
\hline Chinese & 2000 & 2982 & $+982(+33)$ \\
\hline Romance & 1729 & 1759 & $+30(+2)$ \\
\hline Chamic & 1550 & 2672 & $+1,122(+42)$ \\
\hline English-Frisian & 1550 & 1677 & $+127(+8)$ \\
\hline Italo-Western Romance & 1524 & 1600 & $+76(+5)$ \\
\hline Brythonic & 1450 & 1172 & $-278(-24)$ \\
\hline Slavic & 1450 & 1157 & $-293(-25)$ \\
\hline Common Turkic & 1419 & 1348 & $-71(-5)$ \\
\hline Ket-Yugh & 1300 & 970 & $-330(-34)$ \\
\hline Northern Roglai-Tsat & 1000 & 1933 & $+933(+48)$ \\
\hline Maltese-Maghreb Arabic & 910 & 1534 & $+624(+41)$ \\
\hline Western Turkic & 900 & 1076 & $+176(+16)$ \\
\hline East Slavic & 760 & 1288 & $+528(+41)$ \\
\hline Mongolic & 750 & 2267 & $+1,517(+67)$ \\
\hline Romani & 650 & 603 & $-47(-8)$ \\
\hline Maa & 600 & 471 & $-129(-27)$ \\
\hline Southern Songhai & 550 & 580 & $+30(+5)$ \\
\hline Cham & 529 & 781 & $+252(+32)$ \\
\hline Southwest Tungusic & 236 & 832 & $+596(+72)$ \\
\hline \multicolumn{4}{|l|}{ Epigraphic: } \\
\hline Hmong-Mien & 2500 & 4243 & $+1,743(+41)$ \\
\hline Ethiopian Semitic & 2450 & 2408 & $-42(-2)$ \\
\hline Cholan & 1600 & 1148 & $-452(-39)$ \\
\hline Scandinavian & 1100 & 1569 & $+469(+30)$ \\
\hline Czech-Slovak & 1050 & 479 & $-571(-119)$ \\
\hline Goidelic & 1050 & 1837 & $+787(+43)$ \\
\hline Oromo & 460 & 565 & $+105(+19)$ \\
\hline Sorbian & 450 & 443 & $-7(-2)$ \\
\hline
\end{tabular}


written evidence suggesting that Swahili originated along the Kenya coast in and around the Lamu archipelago around 700$900 \mathrm{CE}$. He further notes that "already by the close of the eighth and the start of the ninth century, Swahili merchants had planted settlements as far south along the Indian Ocean coast as northern Mozambique and had apparently reached the Comoro Islands."

Similarity: 50.84 .

Comparisons: Maore (1), Mwani (1), Swahili (8); 17 pairs.

\section{Temotu}

Date: 3200 (A).

Source: In a paper that describes shared linguistic innovations defining a Temotu subgroup within Austronesian, Ross and Næss (2007:461) cite Green (2003) for an archaeological date of about 3200 BP for the first human occupation of the Reef and Santa Cruz islands, which is ascribed to the Lapita culture (correlated with speakers of Austronesian languages) and is said to be among the earliest examples of this culture outside the Bismarck Archipelago.

Similarity: 7.36 .

Comparisons: Reefs-Santa Cruz (7), Utupua-Vanikoro (2); 14 pairs.

\section{Tupi-Guarani (Coastal)}

Date: 1750 (A, H).

Source: Brochado (1984:354) makes reference to ceramic and other archaeological data from Amazonia and adjacent areas as well as ethnohistoric information that together suggest that the ancestors of the Guarani and the ancestors of the Tupinambá evolved independently since 2000-1500 BP.

Similarity: 24.04.

Comparisons: Subgroups I, II (7), Subgroup III (3); 21 pairs. Note: Subgroups are from Brochado (1984).

\section{Turkic (Common Turkic and Chuvash)}

Date: $2500(\mathrm{~A}, \mathrm{H})$.

Source: Róna-Tas (1991:28) correlates Turkic vocabulary with archaeological and historical information to date the beginning of the Late Ancient Turkic period to the middle of the first millennium BCE, stating that "the beginning of the Late [Ancient] Turkic period was marked by the formation of those Turkic dialects which later became the basis for the various groups and single languages" (26).

Similarity: 9.83.

Comparisons: Chuvash (1), Common Turkic (50); 50 pairs. Note: Common Turkic subgroup is from Golden (1998).

\section{Wakashan}

Date: 2500 (A).

Source: Mitchell (1990:357) infers from archaeology that speakers of the Northern branch of Wakashan expanded into the area around Queen Charlotte Strait, probably from the opposite side of Vancouver Island, in about 500 BCE.

Similarity: 14.80 .

Comparisons: Northern (2), Southern (3); 6 pairs.

\section{Western Turkic (Kipchak)}

Date: $900(\mathrm{H})$.

Source: The Kipchak empire spread in the eleventh and twelfth centuries CE (and was destroyed in $1239 \mathrm{CE}$ ), according to Troike (1969:191).

Similarity: 45.36.

Comparisons: Aralo-Caspian (4), Ponto-Caspian (3), Uralian (4); 40 pairs.

This collection of 52 calibration points is substantially larger and more diverse than the 13 points in the calibration of Lees (1953), which has long served as the standard in glottochronology. Lees estimated a constant rate of word replacement by comparing vocabularies of modern languages to those of older language states attested in textual materials. For example, Catalan, French, Italian, Portuguese, Romanian, and Spanish were compared with Latin. The generality of Lees's calibration is limited by the fact that all but two of the 13 languages (Coptic and Mandarin) are Indo-European. The much larger quantitative test of glottochronology by Blust (2000) involves 224 languages, but all belong to a single family, Austronesian. Among the 52 calibration points employed here, 17 are Indo-European and nine are Austronesian, meaning that one-half refer to groups in other families, including languages of Africa, Australia, and North, Middle, and South America as well as Europe, Asia, and Oceania. The geographic distribution of points reflects the distribution of available dates, which is thinnest for Australia, New Guinea, and South America.

\section{Testing ASJP Chronology}

The scatterplot of figure 2 shows the time depth of each calibration group as a function of the average similarity for the group on a logarithmic scale. The correlation (Pearson's $r$ ) between $\log$ similarity and time is -0.84 . To a good approximation, this strong correlation supports the critical claim that log lexical similarity decreases linearly as time depth increases.

The straight line in figure 2 represents formula (3) with

Table 2. Analyses of variance on algebraic and absolute percent discrepancies

\begin{tabular}{lccr}
\hline Factor & $F$ (algebraic) & $F$ (absolute) & df \\
\hline Type of date & 2.52 & .29 & 3,48 \\
Language family & 1.12 & .32 & 16,35 \\
Geographical area & .68 & .87 & 3,48 \\
Mode of subsistence & .43 & .53 & 1,50 \\
\hline
\end{tabular}


Table 3. Automated Similarity Judgment Program (ASJP) dates for language groups of Africa

\begin{tabular}{|c|c|c|c|c|}
\hline Group & Pairs & Subgroups & Similarity & Date \\
\hline Afro-Asiatic & 24,303 & $6(7)$ & 1.77 & 6016 \\
\hline Berber & 139 & $4(4)$ & 29.46 & 1733 \\
\hline Eastern & 2 & $2(2)$ & 30.17 & 1697 \\
\hline Northern & 54 & $3(4)$ & 43.00 & 1158 \\
\hline Tamasheq & 4 & $2(2)$ & 63.83 & 556 \\
\hline Chadic & 2,945 & $4(4)$ & 3.86 & 4826 \\
\hline Biu-Mandara & 44 & $2(3)$ & 4.92 & 4457 \\
\hline Masa & 26 & $6(8)$ & 31.13 & 1649 \\
\hline West & 364 & $2(3)$ & 6.23 & 4099 \\
\hline Cushitic & 752 & $4(4)$ & 4.10 & 4734 \\
\hline Central & 24 & $4(4)$ & 30.38 & 1686 \\
\hline East & 891 & $9(10)$ & 12.44 & 3045 \\
\hline South & 14 & $5(7)$ & 20.20 & 2308 \\
\hline Omotic & 84 & $2(2)$ & 3.52 & 4968 \\
\hline North & 141 & $3(3)$ & 11.71 & 3137 \\
\hline South & 3 & $3(5)$ & 25.34 & 1963 \\
\hline Semitic & 396 & $2(2)$ & 10.51 & 3301 \\
\hline Central & 72 & $2(2)$ & 16.26 & 2638 \\
\hline South & 72 & $2(2)$ & 7.56 & 3804 \\
\hline Khoisan & 31 & $3(3)$ & .01 & 14,592 \\
\hline Southern African Khoisan & 71 & $3(3)$ & 2.88 & 5271 \\
\hline Central & 6 & $2(3)$ & 11.67 & 3143 \\
\hline Northern & 3 & $3(6)$ & 27.36 & 1846 \\
\hline Southern & 4 & $2(2)$ & 5.30 & 4344 \\
\hline Niger-Congo & 51,854 & $4(4)$ & 1.54 & 6227 \\
\hline Atlantic-Congo & 35,936 & $3(3)$ & 1.26 & 6525 \\
\hline Ijoid & 33 & $2(2)$ & 16.87 & 2582 \\
\hline Atlantic & 241 & $3(3)$ & 1.30 & 6480 \\
\hline Northern & 161 & $5(5)$ & 3.32 & 5055 \\
\hline Southern & 9 & $2(3)$ & 4.64 & 4546 \\
\hline Volta-Congo & 54,371 & $5(5)$ & 2.51 & 5484 \\
\hline Benue-Congo & 46,303 & $13(16)$ & 3.58 & 4940 \\
\hline Dogon & 42 & $8(14)$ & 21.65 & 2202 \\
\hline Kru & 6 & $2(5)$ & 20.08 & 2317 \\
\hline Kwa & 216 & $2(2)$ & 5.78 & 4212 \\
\hline Kordofanian & 119 & $4(4)$ & 3.77 & 4861 \\
\hline Heiban & 18 & $2(2)$ & 17.55 & 2521 \\
\hline Katla & 1 & $2(2)$ & 20.72 & 2269 \\
\hline Talodi & 5 & $2(2)$ & 4.31 & 4658 \\
\hline Mande & 799 & $2(2)$ & 9.74 & 3417 \\
\hline Eastern & 70 & $2(2)$ & 26.31 & 1905 \\
\hline Western & 280 & $2(2)$ & 12.42 & 3047 \\
\hline Nilo-Saharan & 7,676 & $10(10)$ & 1.17 & 6642 \\
\hline Central Sudanic & 459 & $2(2)$ & 3.20 & 5114 \\
\hline East & 208 & $4(4)$ & 8.01 & 3715 \\
\hline Eastern Sudanic & 1,091 & $4(4)$ & 1.80 & 5988 \\
\hline Eastern & 57 & $4(4)$ & 3.23 & 5103 \\
\hline Nilotic & 719 & $3(3)$ & 4.76 & 4508 \\
\hline Western & 5 & $2(4)$ & 2.32 & 5601 \\
\hline Kadugli-Krongo & 49 & $6(6)$ & 41.25 & 1221 \\
\hline Komuz & 18 & $2(2)$ & 3.00 & 5209 \\
\hline Koman & 13 & $4(5)$ & 17.31 & 2542 \\
\hline Saharan & 3 & $2(2)$ & 6.91 & 3941 \\
\hline Western & 2 & $2(2)$ & 8.91 & 3553 \\
\hline Songhai & 12 & $2(3)$ & 38.31 & 1333 \\
\hline Northern & 1 & $2(2)$ & 54.15 & 807 \\
\hline Southern & 14 & $5(5)$ & 62.85 & 580 \\
\hline
\end{tabular}

the constants $s_{0}$ and $r$ determined by linear regression of $t$ on $\log s$. The line is chosen to make the most accurate possible predictions of time depth from similarity by minimizing the average of the squared distances on the vertical axis (in years) from the values of $t$ to the line, which are the squared distances between the predicted and observed values of $t$. For this line, $s_{0}=92 \%$ and $r=0.72$ (per 1,000 years). ${ }^{3}$ Specifically, $s_{0}$ is the point where the line crosses the horizontal axis, and $1 /(2 \log r)$ is the slope of the line, which is negative because $r$ is below 1 . The fact that regression analysis produces a value of $s_{0}$ below $100 \%$ is consistent with the usual lexical heterogeneity of languages, including those at time zero.

Table 1 indicates the accuracy of dates based on $s_{0}=$ $92 \%$ and $r=0.72$ for the 52 language groups used for calibration. In table 1 , language groups are categorized by type of calibration date and rank ordered within categories by calibration date from oldest to youngest. The ASJP date is the value of $t$ obtained by substituting the language-group similarity score into formula (3) along with $s_{0}=92 \%$ and $r=0.72$. The next column gives the algebraic difference between the calibration date and the ASJP date, followed in parentheses by this difference as a percentage of the ASJP date.

The algebraic differences between the calibration dates and the ASJP dates include a positive or negative sign and thus show whether the ASJP dates are respectively greater or less than the calibration dates. As in any linear regression, the algebraic differences have a mean of 0 and a correlation of 0 with the ASJP dates (within rounding error), meaning that the ASJP dates are unbiased. The absolute differences disregard sign and thus show how much the ASJP dates depart from the calibration dates in either direction. Absolute differences have a correlation of 0.57 with the ASJP dates and tend to be larger for older than for younger calibration dates, indicating that older ASJP dates are less accurate than younger ones.

The absolute percentage differences have a correlation of only -0.06 with the ASJP dates, indicating that the discrepancies in ASJP dates are approximately proportional to the dates themselves. The mean absolute percent discrepancy is $29 \%$; of the 52 ASJP dates, five are off by more than $50 \%$, and one is off by more than $100 \%$.

Although the calibration dates older than $2500 \mathrm{BP}$ are all archaeological, figure 2 shows that younger dates of all four types are about equally close to the regression line. To deter-

3. Although $92 \%$ for $s_{0}$ and 0.72 for $r$ are the values that make the best predictions of time depth, they are not the only values consistent with the calibration data. The regression with $s_{0}=92 \%$ and $r=0.72$ is based on the assumption that all the error is in the dates. The alternative assumption that all the error is in the similarities produces $s_{0}=62 \%$ and $r=0.79$, while intermediate distributions of error produce intermediate values of $s_{0}$ and $r$. These regression analyses imply the testable prediction that if independent estimates of $s_{0}$ and $r$ are derived from other data, they should be between $62 \%$ and $92 \%$ for $s_{0}$ and between 0.72 and 0.79 for $r$. 
Table 4. Automated Similarity Judgment Program (ASJP) dates for language groups of Eurasia

\begin{tabular}{|c|c|c|c|c|}
\hline Group & Pairs & Subgroups & Similarity & Date \\
\hline Altaic & 1,588 & $3(3)$ & 1.84 & 5954 \\
\hline Mongolic & 7 & $2(2)$ & 20.75 & 2267 \\
\hline Eastern & 16 & $3(3)$ & 22.48 & 2145 \\
\hline Tungusic & 99 & $2(2)$ & 38.67 & 1319 \\
\hline Northern & 20 & $3(3)$ & 44.90 & 1092 \\
\hline Southern & 24 & $2(2)$ & 32.26 & 1595 \\
\hline Turkic & 50 & $2(2)$ & 9.83 & 3404 \\
\hline Common & 713 & $4(7)$ & 37.94 & 1348 \\
\hline Andamanese & 16 & $2(2)$ & 4.75 & 4510 \\
\hline Great Andamanese & 12 & $2(2)$ & 22.82 & 2122 \\
\hline South Andamanese & 1 & $2(3)$ & 42.22 & 1186 \\
\hline Austro-Asiatic & 1,843 & $2(2)$ & 8.45 & 3635 \\
\hline Mon-Khmer & 3,358 & $8(9)$ & 9.81 & 3406 \\
\hline Aslian & 29 & $4(4)$ & 23.46 & 2080 \\
\hline Eastern Mon-Khmer & 475 & $4(4)$ & 18.05 & 2479 \\
\hline Nicobar & 3 & $3(5)$ & 11.56 & 3158 \\
\hline Northern Mon-Khmer & 243 & $4(4)$ & 10.81 & 3259 \\
\hline Palyu & 1 & $2(2)$ & 14.05 & 2861 \\
\hline Viet-Muong & 22 & $4(5)$ & 20.45 & 2289 \\
\hline Munda & 60 & $2(2)$ & 16.96 & 2574 \\
\hline North Munda & 14 & $2(2)$ & 41.58 & 1209 \\
\hline South Munda & 4 & $2(2)$ & 17.69 & 2510 \\
\hline Chukotko-Kamchatkan & 6 & $2(2)$ & 10.06 & 3368 \\
\hline Northern Chukotko-Kamchatkan & 2 & $2(2)$ & 42.04 & 1192 \\
\hline Dravidian & 181 & $4(10)$ & 23.84 & 2055 \\
\hline Central & 2 & $2(2)$ & 58.29 & 695 \\
\hline Northern & 3 & $3(5)$ & 24.24 & 2030 \\
\hline South-Central & 6 & $2(2)$ & 18.43 & 2447 \\
\hline Southern & 9 & $2(10)$ & 26.50 & 1894 \\
\hline Hmong-Mien & 49 & $3(3)$ & 5.66 & 4243 \\
\hline Hmongic & 21 & $4(5)$ & 14.84 & 2777 \\
\hline Indo-European & 12,264 & $9(9)$ & 5.29 & 4348 \\
\hline Baltic & $\ldots$ & $\ldots$ & $\ldots$ & $\ldots$ \\
\hline Eastern & 1 & $2(2)$ & 35.05 & 1469 \\
\hline Celtic & $\ldots$ & $\ldots$ & $\ldots$ & $\ldots$ \\
\hline Insular & 6 & $2(2)$ & 7.21 & 3876 \\
\hline Germanic & 161 & $2(3)$ & 29.24 & 1745 \\
\hline North & 10 & $2(2)$ & 32.83 & 1569 \\
\hline West & 170 & $4(4)$ & 36.71 & 1398 \\
\hline Indo-Iranian & 5,022 & $2(2)$ & 8.28 & 3665 \\
\hline Indo-Aryan & 2,586 & $8(11)$ & 24.79 & 1996 \\
\hline Iranian & 329 & $2(4)$ & 14.09 & 2856 \\
\hline Italic & $\ldots$ & $\ldots$ & $\ldots$ & $\ldots$ \\
\hline Romance & 24 & $2(3)$ & 28.96 & 1759 \\
\hline Slavic & 84 & $3(3)$ & 43.01 & 1157 \\
\hline East & 5 & $3(4)$ & 39.47 & 1288 \\
\hline South & 8 & $2(2)$ & 58.44 & 691 \\
\hline West & 11 & $3(3)$ & 53.67 & 820 \\
\hline Japonic & 12 & $2(2)$ & 32.92 & 1564 \\
\hline Kartvelian & 5 & $3(3)$ & 12.82 & 2999 \\
\hline Zan & 1 & $2(2)$ & 62.20 & 596 \\
\hline North Caucasian & 160 & $2(2)$ & .58 & 7709 \\
\hline East Caucasian & 391 & $7(7)$ & 7.06 & 3907 \\
\hline West Caucasian & 8 & $3(3)$ & 8.37 & 3649 \\
\hline Sino-Tibetan & 1,106 & $2(2)$ & 2.90 & 5261 \\
\hline Chinese & 19 & $5(14)$ & 12.97 & 2982 \\
\hline Tibeto-Burman & 10,352 & $14(18)$ & 5.81 & 4203 \\
\hline Bai & 107 & $3(3)$ & 34.49 & 1494 \\
\hline Himalayish & 680 & $2(3)$ & 11.37 & 3182 \\
\hline Karen & 9 & $2(4)$ & 19.71 & 2345 \\
\hline Kuki-Chin-Naga & 65 & $2(2)$ & 9.79 & 3411 \\
\hline Lolo-Burmese & 24 & $3(4)$ & 9.63 & 3436 \\
\hline
\end{tabular}

This content downloaded from \$54.094.206.016 on May 04, 2017 03:58:24 AM

All use subject to University of Chicago Press Terms and Conditions (http://www.journals.uchicago.edu/t-and-c). 
Table 4 (Continued)

\begin{tabular}{lrrrr}
\hline Group & Pairs & Subgroups & Similarity & Date \\
\hline Nungish & 2 & $2(5)$ & 25.46 & 1955 \\
$\quad$ Tangut-Qiang & 2 & $2(2)$ & 4.31 & 4660 \\
Tai-Kadai & 699 & $3(3)$ & 10.86 & 3252 \\
Hlai & 2 & $2(2)$ & 19.61 & 2353 \\
Kadai & 24 & $3(3)$ & 16.53 & 2613 \\
Kam-Tai & 505 & $3(3)$ & 19.31 & 2376 \\
Uralic & 240 & $9(9)$ & 11.40 & 3178 \\
Finnic & 14 & $5(11)$ & 51.75 & 876 \\
Mordvin & 1 & $2(2)$ & 54.39 & 800 \\
Permian & 2 & $2(2)$ & 49.17 & 953 \\
Sami & 9 & $2(3)$ & 33.63 & 1532 \\
Samoyed & 1 & $2(5)$ & 14.14 & 2850 \\
Yeniseian & 12 & $1(1)$ & 16.01 & 2661 \\
Assan-Kott & 1 & +2 & 55.07 & 781 \\
Awin-Pumpokol & 1 & +2 & 14.99 & 2762 \\
Ket-Yugh & 1 & $2(2)$ & 48.65 & 970 \\
Yukaghir & 1 & $2(2)$ & 24.28 & 2027 \\
\hline
\end{tabular}

mine whether differences nevertheless exist among the types of dates, the standard statistical test is a one-way analysis of variance (ANOVA) in which the variance between groups of scores is compared with the variance of scores within groups. The test statistic, $F$, is a ratio of variances with degrees of freedom that depend on the number of groups and the number of individual scores. Under the null hypothesis of no differences between groups, the expected value of $F$ is 1 , and values of $F$ significantly above 1 indicate significant differences among the groups. A significance criterion of $P<.05$ is used in all tests reported here.

Table 2 gives the results of ANOVAs for type of date and other possibly relevant factors. The second and third columns show the values of $F$ obtained for differences in algebraic and absolute percent discrepancies, respectively, and the fourth column shows the degrees of freedom. If some types of dates are biased low or high relative to others, there would be differences in the algebraic percent discrepancies, and if some types of dates are more accurate than others, there would be differences in the absolute percent discrepancies. The values of $F$ in the first row of table 2 (for type of date) are not significant, confirming the impression from figure 2 that the types of date do not differ in bias or accuracy.

The 52 calibration points pertain to 17 language families. Families whose languages change rapidly would produce ASJP dates older than the calibrations, and families with low change rates would produce younger dates. Differences among families in the variability of the rate of lexical change would be reflected in the absolute percent discrepancies. The nonsignificant $F$ values in the second row of table 2 suggest no differences among families in the rate or variability of lexical change. To test whether the conditions in different geographical areas influence rates of lexical change, the calibration points are sorted geographically into areas defined as in tables 3-7: Africa (11 points), Eurasia (26 points), the Pacific area (10 points), and the Americas ( 5 points). The third row shows no significant differences between geographical areas. To test the effect of mode of subsistence, Hammarström's (2010) compilation is used to categorize the calibration points according to whether their languages are spoken in predominantly agricultural societies (45 points) or in foraging and pastoral societies ( 7 points: Central Southern African Khoisan, Inuit, Ket-Yugh, Mississippi Valley Siouan, Pama-Nyungan, Saami, and Wakashan). The last row again shows no significant differences.

In summary, basic vocabulary changes at a sufficiently constant rate to produce a robust correlation of -0.84 between $\log$ similarity and calibration date, and the resulting ASJP dates are impervious to all the extraneous factors tested. The observed discrepancies are perhaps even overestimates because they reflect not only variation in the rate of lexical change but also difficulties encountered in matching dated events with linguistic divergences as well as uncertainty regarding the calibration dates themselves, some of which are expressed in the sources as ranges of possible dates. The $29 \%$ mean absolute discrepancy thus represents an upper bound on the expected discrepancy between ASJP dates and true dates.

A possible theoretical framework for the present results can be found in Dixon (1997). Dixon discusses a model of language change involving "punctuated equilibrium," in which languages usually change at a steady rate but occasionally undergo periods of rapid change caused by external events such as natural disasters, material innovations, development of aggressive tendencies, and so on. In Dixon's model, a few languages may undergo more periods of equilibrium and fewer bouts of punctuation (or vice versa) than are typical. As examples of these situations, Bergsland and Vogt (1962) report unusually low rates of lexical change in Icelandic, Georgian, and Armenian and an unusually high rate of change in East Greenlandic Eskimo. However, for most languages, relative amounts of equilibrium and punctuation are more 
Table 5. Automated Similarity Judgment Program (ASJP) dates for language groups of the Pacific

\begin{tabular}{|c|c|c|c|c|}
\hline Group & Pairs & Subgroups & Similarity & Date \\
\hline Amto-Musan & 2 & $2(2)$ & 21.84 & 2189 \\
\hline Arai-Kwomtari & 20 & $2(2)$ & .72 & 7386 \\
\hline Arai (Left May) & 6 & $4(4)$ & 13.04 & 2974 \\
\hline Kwomtari & 9 & $4(4)$ & 1.82 & 5968 \\
\hline Australian & 10,664 & $16(16)$ & 2.84 & 5296 \\
\hline Bunaban & 1 & $2(2)$ & 33.50 & 1538 \\
\hline Daly & 95 & $3(4)$ & 6.91 & 3941 \\
\hline Bringen-Wagaydy & 25 & $2(2)$ & 20.04 & 2320 \\
\hline Malagmalag & 6 & $2(2)$ & 31.43 & 1635 \\
\hline Murrinh-Patha & 2 & $2(2)$ & 15.14 & 2747 \\
\hline Djeragan & 1 & $2(3)$ & 15.10 & 2750 \\
\hline Giimbiyu & 3 & $3(3)$ & 70.03 & 415 \\
\hline Gunwingguan & 274 & $12(13)$ & 4.73 & 4517 \\
\hline Burarran & 3 & $3(4)$ & 8.57 & 3612 \\
\hline Enindhilyagwa & 3 & $3(3)$ & 4.07 & 4746 \\
\hline Gunwinggic & 1 & $2(2)$ & 13.24 & 2951 \\
\hline Maran & 2 & $2(2)$ & 16.02 & 2661 \\
\hline Rembargic & 1 & $2(2)$ & 25.97 & 1925 \\
\hline Yangmanic & 1 & $2(3)$ & 31.97 & 1609 \\
\hline Pama-Nyungan & 6,693 & $27(30)$ & 5.47 & 4295 \\
\hline Arandic & 9 & $4(6)$ & 26.54 & 1892 \\
\hline Dyirbalic & 6 & $3(3)+1$ & 22.60 & 2137 \\
\hline Galgadungic & 1 & $2(2)$ & 19.44 & 2366 \\
\hline Karnic & 13 & $3(3)+1$ & 14.13 & 2851 \\
\hline Maric & 35 & $8(12)$ & 49.98 & 929 \\
\hline Paman & 168 & $7(15)$ & 3.64 & 4918 \\
\hline South-West & 228 & $11(17)$ & 11.98 & 3103 \\
\hline Waka-Kabic & 5 & $3(3)$ & 20.70 & 2270 \\
\hline Wiradhuric & 3 & $3(3)$ & 43.82 & 1129 \\
\hline Worimi & 1 & $2(2)$ & 18.12 & 2473 \\
\hline Yidinic & 1 & $2(2)$ & 40.81 & 1237 \\
\hline Yuin & 3 & $2(2)+1$ & 34.27 & 1503 \\
\hline Yuulngu & 76 & $3(3)$ & 33.13 & 1555 \\
\hline West Barkly & 3 & $3(3)$ & 16.34 & 2631 \\
\hline Wororan & 13 & $4(7)$ & 21.93 & 2183 \\
\hline Yiwaidjan & 5 & $3(3)$ & 13.85 & 2882 \\
\hline Yiwaidjic & 1 & $2(5)$ & 36.50 & 1407 \\
\hline Austronesian & 19,212 & $10(11)$ & 8.46 & 3633 \\
\hline Atayalic & 1 & $2(2)$ & 15.98 & 2664 \\
\hline East Formosan & 5 & $3(3)$ & 19.11 & 2392 \\
\hline Malayo-Polynesian & 268,972 & $16(17)$ & 12.62 & 3024 \\
\hline Celebic & 814 & $4(4)$ & 28.27 & 1796 \\
\hline Eastern & 43 & $2(2)$ & 29.92 & 1710 \\
\hline Kaili-Pamona & 2 & $2(2)$ & 45.36 & 1076 \\
\hline Tomini-Tolitoli & 20 & $2(2)$ & 35.07 & 1468 \\
\hline Central-Eastern & 51,447 & $3(4)$ & 11.92 & 3111 \\
\hline Central Malayo-Polynesian & 4,562 & $9(10)$ & 18.82 & 2415 \\
\hline Eastern Malayo-Polynesian & 18,404 & $2(2)$ & 7.56 & 3803 \\
\hline Greater Barito & 1,012 & $4(4)$ & 24.23 & 2031 \\
\hline East & 156 & $3(3)$ & 26.73 & 1881 \\
\hline Sama-Bajaw & 21 & $2(2)$ & 34.59 & 1489 \\
\hline West & 15 & $2(2)$ & 45.03 & 1087 \\
\hline Javanese & 2 & $2(5)$ & 63.45 & 566 \\
\hline Lampung & 164 & $3(3)$ & 54.92 & 785 \\
\hline Land Dayak & 3 & $3(5)$ & 34.11 & 1510 \\
\hline Malayo-Sumbawan & 65 & $3(3)$ & 27.37 & 1845 \\
\hline North and East & 221 & $3(3)$ & 26.44 & 1898 \\
\hline North Borneo & 80 & $3(5)$ & 24.46 & 2016 \\
\hline Melanau-Kajang & 1 & $2(2)$ & 37.34 & 1372 \\
\hline North Sarawakan & 33 & $4(5)$ & 22.08 & 2172 \\
\hline Sabahan & 7 & $3(3)$ & 38.32 & 1333 \\
\hline Northwest Sumatra-Barrier Islands & 5 & $3(5)$ & 27.79 & 1822 \\
\hline Philippine & 7,587 & $8(10)$ & 27.65 & 1830 \\
\hline Bashiic & 9 & $2(2)$ & 57.43 & 717 \\
\hline
\end{tabular}

This content downloaded from \$59.094.206.016 on May 04, 2017 03:58:24 AM

All use subject to University of Chicago Press Terms and Conditions (http://www.journals.uchicago.edu/t-and-c). 
Table 5 (Continued)

\begin{tabular}{|c|c|c|c|c|}
\hline Group & Pairs & Subgroups & Similarity & Date \\
\hline Bilic & 19 & $3(4)$ & 31.46 & 1633 \\
\hline Central Luzon & 2 & $2(3)$ & 40.42 & 1252 \\
\hline Greater Central Philippine & 1,949 & $8(8)$ & 38.49 & 1326 \\
\hline Minahasan & 4 & $2(2)$ & 61.87 & 604 \\
\hline Northern Luzon & 419 & $3(4)$ & 31.72 & 1621 \\
\hline Sangiric & 5 & $2(2)$ & 66.95 & 484 \\
\hline South Sulawesi & 39 & $3(5)$ & 48.63 & 970 \\
\hline Bugis & 2 & $2(3)$ & 51.47 & 884 \\
\hline Makassar & 8 & $3(5)$ & 63.78 & 558 \\
\hline Northern & 3 & $3(17)$ & 73.36 & 345 \\
\hline Northwest Formosan & 1 & $2(2)$ & 21.63 & 2204 \\
\hline Tsouic & 3 & $3(3)$ & 20.42 & 2291 \\
\hline Western Plains & 3 & $2(2)$ & 16.83 & 2586 \\
\hline Central Western Plains & 2 & $2(2)$ & 18.63 & 2431 \\
\hline Border & 64 & $2(3)$ & 9.51 & 3453 \\
\hline Taikat & 15 & $2(2)$ & 18.96 & 2404 \\
\hline Waris & 24 & $5(8)$ & 20.83 & 2261 \\
\hline Central Solomons & 9 & $4(4)$ & 8.21 & 3677 \\
\hline East Bird's Head-Sentani & 39 & $3(3)$ & 1.19 & 6615 \\
\hline East Bird's Head & 2 & $2(2)$ & 8.70 & 3590 \\
\hline Sentani & 20 & $2(2)$ & 6.22 & 4101 \\
\hline East Geelvink Bay & 4 & $2(10)$ & 6.73 & 3979 \\
\hline Eastern Trans-Fly & 495 & $4(4)$ & 10.83 & 3257 \\
\hline \multicolumn{5}{|l|}{ Kaure } \\
\hline Kaure Proper & 1 & $2(3)$ & 15.98 & 2665 \\
\hline Lakes Plain & 93 & $4(4)$ & 2.87 & 5279 \\
\hline Rasawa-Saponi & 1 & $2(2)$ & 12.51 & 3037 \\
\hline Tariku & 149 & $4(4)$ & 8.98 & 3541 \\
\hline Left May & 2 & $2(2)$ & 15.98 & 2665 \\
\hline Mairasi & 4 & $2(3)$ & 41.93 & 1196 \\
\hline Nimboran & 6 & $2(5)$ & 23.78 & 2059 \\
\hline North Bougainville & 1 & $2(3)$ & 13.46 & 2925 \\
\hline Pauwasi & 12 & $2(2)$ & 6.21 & 4102 \\
\hline Eastern & 2 & $2(3)$ & 14.22 & 2842 \\
\hline Western & 3 & $2(2)$ & 28.69 & 1774 \\
\hline Piawi & 10 & $2(2)$ & 11.22 & 3203 \\
\hline Ramu-Lower Sepik & 117 & $3(3)$ & .96 & 6942 \\
\hline Lower Sepik & 28 & $4(4)$ & 9.78 & 3411 \\
\hline Ramu & 28 & $4(6)$ & 6.65 & 4000 \\
\hline Sepik & 294 & $8(12)$ & 3.86 & 4827 \\
\hline Ndu & 32 & $6(12)$ & 41.08 & 1227 \\
\hline Nukuma & 1 & $2(3)$ & 28.37 & 1791 \\
\hline Ram & 2 & $2(3)$ & 28.35 & 1791 \\
\hline Sepik Hill & 29 & $4(4)$ & 9.00 & 3538 \\
\hline Sko & 48 & $2(2)$ & 4.85 & 4478 \\
\hline Krisa & 27 & $3(4)+4$ & 19.01 & 2400 \\
\hline Vanimo & 14 & $3(3)+2$ & 28.24 & 1798 \\
\hline South Bougainville & 2 & $2(2)$ & 12.37 & 3054 \\
\hline Buin & 1 & $2(3)$ & 29.25 & 1744 \\
\hline South-Central Papuan & 145 & $4(4)$ & 1.53 & 6232 \\
\hline Morehead-Upper Maro & 14 & $3(3)$ & 2.73 & 5353 \\
\hline Pahoturi & 5 & $2(2)$ & 24.02 & 2044 \\
\hline Yelmek-Maklew & 4 & $2(2)$ & 35.06 & 1468 \\
\hline Tor-Kwerba & 45 & $2(2)$ & 4.99 & 4435 \\
\hline Greater Kwerba & 20 & $3(3)$ & 6.18 & 4109 \\
\hline Kwerba & 8 & $2(2)$ & 7.32 & 3852 \\
\hline Orya-Tor & 6 & $2(3)$ & 8.13 & 3693 \\
\hline Torricelli & 250 & $6(7)$ & 2.10 & 5754 \\
\hline Kombio-Arapesh & 12 & $2(2)$ & 10.15 & 3356 \\
\hline Marienberg & 34 & $7(7)$ & 10.25 & 3339 \\
\hline Momumbo & 1 & $2(2)$ & 26.98 & 1867 \\
\hline Wapei-Palei & 7 & $3(3)$ & 2.67 & 5386 \\
\hline Trans-New Guinea & 77,005 & $39(39)$ & 1.20 & 6609 \\
\hline Angan & $\cdots$ & & $\ldots$ & $\ldots$ \\
\hline Nuclear Angan & 1 & $2(12)$ & 4.71 & 4523 \\
\hline
\end{tabular}

This content downloaded from 854.094.206.016 on May 04, 2017 03:58:24 AM

All use subject to University of Chicago Press Terms and Conditions (http://www.journals.uchicago.edu/t-and-c). 
Table 5 (Continued)

\begin{tabular}{|c|c|c|c|c|}
\hline Group & Pairs & Subgroups & Similarity & Date \\
\hline Asmat-Kamoro & 21 & $4(5)$ & 21.83 & 2189 \\
\hline Asmat & 6 & $4(6)$ & 46.66 & 1033 \\
\hline Sabakor & 1 & $2(2)$ & 63.37 & 567 \\
\hline Binanderean & $\cdots$ & $\cdots$ & $\cdots$ & $\cdots$ \\
\hline Binandere & 9 & $4(12)$ & 27.42 & 1842 \\
\hline Bosavi & 96 & $8(9)$ & 19.66 & 2349 \\
\hline Chimbu-Wahgi & 31 & $3(4)$ & 9.41 & 3470 \\
\hline Chimbu & 9 & $4(7)$ & 31.42 & 1635 \\
\hline Hagen & 2 & $2(2)$ & 34.22 & 1505 \\
\hline Jimi & 1 & $2(3)$ & 50.55 & 912 \\
\hline Duna-Bogaya & 1 & $2(2)$ & 12.78 & 3004 \\
\hline East Strickland & 18 & $5(6)$ & 36.66 & 1401 \\
\hline Eleman & 20 & $3(3)$ & 3.80 & 4851 \\
\hline Nuclear Eleman & 8 & $2(2)$ & 40.31 & 1256 \\
\hline Engan & 56 & $3(3)$ & 14.99 & 2762 \\
\hline Enga & 24 & $4(6)$ & 18.94 & 2406 \\
\hline Angal-Kewa & 5 & $3(7)$ & 33.12 & 1555 \\
\hline Finisterre-Huon & 70 & $2(2)$ & 6.08 & 4136 \\
\hline Finisterre & 9 & $4(6)$ & 13.98 & 2868 \\
\hline Huon & 48 & $2(3)$ & 12.45 & 3044 \\
\hline Gogodala-Suki & 7 & $2(2)$ & 14.36 & 2827 \\
\hline Gogodala & 6 & $2(3)$ & 34.47 & 1494 \\
\hline Inland Gulf & 2 & $2(2)$ & 13.99 & 2867 \\
\hline Minanibai & 1 & $2(6)$ & 21.72 & 2197 \\
\hline Kainantu-Goroka & 140 & $2(2)$ & 3.81 & 4847 \\
\hline Gorokan & 76 & $6(6)$ & 11.34 & 3186 \\
\hline Kainantu & 20 & $2(5)$ & 11.96 & 3105 \\
\hline Kayagar & 5 & $3(3)$ & 39.54 & 1285 \\
\hline Kiwaian & 88 & $7(7)$ & 35.80 & 1436 \\
\hline Kolopom & 3 & $3(3)$ & 13.76 & 2892 \\
\hline Madang & 3,025 & $4(4)$ & 4.56 & 4573 \\
\hline Croisilles & 1,082 & $7(7)$ & 6.19 & 4107 \\
\hline Rai Coast & 378 & $7(7)$ & 9.16 & 3511 \\
\hline South Adelbert Range & 66 & $3(3)$ & 5.96 & 4165 \\
\hline Marind & 57 & $3(3)$ & 6.58 & 4014 \\
\hline Boazi & 12 & $2(2)$ & 32.22 & 1597 \\
\hline Yaqay & 2 & $2(2)$ & 23.63 & 2069 \\
\hline Mek & 3 & $2(2)$ & 38.92 & 1309 \\
\hline Eastern & 3 & $3(6)$ & 36.08 & 1425 \\
\hline Mombum & 1 & $2(2)$ & 38.82 & 1313 \\
\hline Ok-Awyu & 108 & $2(2)$ & 5.56 & 4272 \\
\hline Awyu-Dumut & 27 & $4(4)$ & 13.55 & 2916 \\
\hline Ok & 36 & $2(5)$ & 17.41 & 2534 \\
\hline South Bird's Head & 69 & $3(3)$ & 8.86 & 3561 \\
\hline South Bird's Head Proper & 40 & $3(3)$ & 32.52 & 1583 \\
\hline Southeast Papuan & 268 & $6(7)$ & 2.85 & 5286 \\
\hline Kwalean & 11 & $3(3)$ & 12.55 & 3032 \\
\hline Goilalan & 2 & $2(2)$ & 5.70 & 4233 \\
\hline Koiarian & 12 & $2(2)$ & 15.70 & 2691 \\
\hline Mailuan & 3 & $3(6)$ & 40.79 & 1238 \\
\hline Manubaran & 8 & $2(2)$ & 45.70 & 1065 \\
\hline Teberan & 1 & $2(2)$ & 20.00 & 2322 \\
\hline Turama-Kikorian & 3 & $2(2)$ & 12.58 & 3028 \\
\hline Turama-Omatian & 2 & $2(2)$ & 32.59 & 1580 \\
\hline West & 886 & $5(5)$ & 3.26 & 5082 \\
\hline Dani & 20 & $3(3)$ & 28.53 & 1782 \\
\hline East Timor & 2 & $2(2)$ & 26.13 & 1916 \\
\hline West Bomberai & 2 & $2(2)$ & 9.25 & 3497 \\
\hline West Timor-Alor-Pantar & 114 & $4(5)$ & 9.04 & 3531 \\
\hline Wissel Lakes & 3 & $3(5)$ & 23.77 & 2060 \\
\hline West Papuan & 324 & $3(3)$ & .24 & 9083 \\
\hline North Halmahera & 97 & $4(4)$ & 13.14 & 2962 \\
\hline West Bird's Head & 31 & $5(5)$ & 17.66 & 2512 \\
\hline Yele-West New Britain & 1 & $2(2)$ & 1.47 & 6293 \\
\hline
\end{tabular}

This content downloaded from $\$ 59$ 4.094.206.016 on May 04, 2017 03:58:24 AM

All use subject to University of Chicago Press Terms and Conditions (http://www.journals.uchicago.edu/t-and-c). 
Table 6. Automated Similarity Judgment Program (ASJP) dates for language groups of North and Middle America

\begin{tabular}{|c|c|c|c|c|}
\hline Group & Pairs & Subgroups & Similarity & Date \\
\hline Algic & 51 & $3(3)$ & 2.39 & 5554 \\
\hline Algonquian & 180 & $4(7)$ & 10.23 & 3343 \\
\hline Central & 77 & $7(8)$ & 15.84 & 2678 \\
\hline Eastern & 28 & $8(10)$ & 12.60 & 3026 \\
\hline Plains & 1 & $2(3)$ & 3.44 & 5002 \\
\hline Caddoan & 3 & $2(2)$ & 3.86 & 4828 \\
\hline Northern & 2 & $2(2)$ & 12.52 & 3035 \\
\hline Chumash & 10 & $5(7)$ & 28.34 & 1792 \\
\hline Eskimo-Aleut & 8 & $2(2)$ & 3.26 & 5084 \\
\hline Eskimo & 16 & $2(2)$ & 27.43 & 1842 \\
\hline Gulf & 3 & $3(4)$ & .53 & 7859 \\
\hline Hokan & 167 & $3(3)$ & 3.64 & 4915 \\
\hline Esselen-Yuman & $\ldots$ & $\ldots$ & $\ldots$ & $\ldots$ \\
\hline Yuman & 46 & $5(6)$ & 27.01 & 1865 \\
\hline Northern & 47 & $3(3)$ & 2.22 & 5666 \\
\hline Karok-Shasta & 4 & $2(2)$ & 2.93 & 5246 \\
\hline Pomo & 6 & $2(2)$ & 41.12 & 1226 \\
\hline Iroquoian & 6 & $2(2)$ & 3.79 & 4855 \\
\hline Northern Iroquoian & 5 & $2(3)$ & 11.42 & 3176 \\
\hline Five Nations & 6 & $2(2)$ & 30.64 & 1673 \\
\hline Kiowa-Tanoan & 2 & $2(2)$ & 9.64 & 3434 \\
\hline Mayan & 1,449 & $5(5)$ & 21.39 & 2220 \\
\hline Cholan-Tzeltalan & 20 & $2(2)$ & 35.91 & 1432 \\
\hline Cholan & 6 & $2(2)$ & 43.26 & 1148 \\
\hline Tzeltalan & 6 & $4(8)$ & 65.76 & 511 \\
\hline Huastecan & 1 & $2(4)$ & 40.29 & 1257 \\
\hline Kanjobalan-Chujean & 15 & $2(2)$ & 41.14 & 1225 \\
\hline Chujean & 2 & $2(3)$ & 45.89 & 1058 \\
\hline Kanjobalan & 4 & $2(2)$ & 54.27 & 803 \\
\hline Quichean-Mamean & 667 & $2(2)$ & 31.14 & 1649 \\
\hline Greater Mamean & 100 & $2(2)$ & 34.51 & 1492 \\
\hline Greater Quichean & 166 & $6(6)$ & 48.30 & 981 \\
\hline Yucatecan & 6 & $2(2)$ & 54.76 & 790 \\
\hline Mopan-Itza & 2 & $2(2)$ & 51.35 & 887 \\
\hline Yucatec-Lacandon & 1 & $2(3)$ & 62.01 & 601 \\
\hline Misumalpan & 3 & $3(4)$ & 14.87 & 2774 \\
\hline Mixe-Zoque & 49 & $2(2)$ & 36.51 & 1407 \\
\hline Mixe & 14 & $3(3)$ & 50.94 & 900 \\
\hline Zoque & 16 & $3(3)$ & 54.86 & 787 \\
\hline Muskogean & 8 & $2(2)$ & 29.71 & 1720 \\
\hline Eastern & 6 & $4(4)$ & 42.16 & 1188 \\
\hline Western & 1 & $2(2)$ & 73.34 & 345 \\
\hline Na-Dene & 22 & $2(2)$ & $-.25^{\mathrm{a}}$ & $\ldots$ \\
\hline Nuclear Na-Dene & 21 & $2(2)$ & .34 & 8532 \\
\hline Athapaskan-Eyak & 20 & $2(2)$ & 5.82 & 4203 \\
\hline Athapaskan & 138 & $4(8)$ & 23.74 & 2062 \\
\hline Oto-Manguean & 2,108 & $8(8)$ & 1.21 & 6591 \\
\hline Chiapanec-Mangue & 1 & $1(1)+1$ & 18.46 & 2445 \\
\hline Chinantecan & 6 & $4(14)$ & 25.80 & 1935 \\
\hline Mixtecan & 14 & $2(2)$ & 4.65 & 4542 \\
\hline Mixtec-Cuicatec & 6 & $2(2)$ & 11.69 & 3140 \\
\hline Trique & 1 & $2(3)$ & 46.96 & 1024 \\
\hline Otopamean & 10 & $2(4)$ & 8.34 & 3654 \\
\hline Otomian & 6 & $2(2)$ & 21.48 & 2214 \\
\hline Popolocan & 71 & $3(3)$ & 12.52 & 3036 \\
\hline Chocho-Popolocan & 6 & $2(2)$ & 21.55 & 2209 \\
\hline Mazatecan & 52 & $8(8)$ & 55.29 & 775 \\
\hline Subtiaba-Tlapanecan & 14 & $5(5)$ & 49.35 & 948 \\
\hline Zapotecan & 75 & $2(2)$ & 11.62 & 3149 \\
\hline Chatino & 3 & $3(6)$ & 47.79 & 997 \\
\hline
\end{tabular}

This content downloaded from \$59.094.206.016 on May 04, 2017 03:58:24 AM

All use subject to University of Chicago Press Terms and Conditions (http://www.journals.uchicago.edu/t-and-c). 
Table 6 (Continued)

\begin{tabular}{|c|c|c|c|c|}
\hline Group & Pairs & Subgroups & Similarity & Date \\
\hline Zapotec & 300 & $25(57)$ & 30.60 & 1676 \\
\hline Penutian & 230 & $7(8)$ & 2.44 & 5522 \\
\hline Maiduan & 5 & $3(4)$ & 41.29 & 1219 \\
\hline Oregon Penutian & 3 & $2(3)$ & .04 & 11,886 \\
\hline Coast Oregon & 3 & $3(3)$ & 3.67 & 4902 \\
\hline Plateau Penutian & 2 & $2(2)$ & 6.03 & 4147 \\
\hline Sahaptin & 1 & $2(5)$ & 15.35 & 2725 \\
\hline Yok-Utian & 18 & $2(2)$ & 5.07 & 4413 \\
\hline Utian & 14 & $2(2)$ & 8.29 & 3663 \\
\hline Miwokan & 12 & $2(2)$ & 22.54 & 2141 \\
\hline Salishan & 129 & $5(5)$ & 7.44 & 3827 \\
\hline Central Salish & 37 & $5(6)$ & 18.29 & 2459 \\
\hline Interior Salish & 8 & $2(2)$ & 12.98 & 2980 \\
\hline Siouan & 15 & $2(2)$ & 1.59 & 6178 \\
\hline Siouan Proper & 56 & $3(3)$ & 11.47 & 3169 \\
\hline Tequistlatecan & 1 & $2(2)$ & 41.50 & 1212 \\
\hline Totonacan & 4 & $2(2)$ & 35.83 & 1435 \\
\hline Totonac & 48 & $6(9)+1$ & 65.96 & 506 \\
\hline Tepehua & 3 & $3(3)$ & 64.26 & 546 \\
\hline Uto-Aztecan & 781 & $2(2)$ & 6.56 & 4018 \\
\hline Northern Uto-Aztecan & 33 & $4(4)$ & 16.93 & 2576 \\
\hline Numic & 14 & $3(3)$ & 29.40 & 1737 \\
\hline Southern Uto-Aztecan & 754 & $2(2)$ & 9.40 & 3472 \\
\hline Sonoran & 61 & $4(5)$ & 19.01 & 2400 \\
\hline Aztecan & $\ldots$ & $\ldots$ & $\ldots$ & $\ldots$ \\
\hline General Aztec & 57 & $2(2)$ & 34.13 & 1509 \\
\hline Wakashan & 6 & $2(2)$ & 14.80 & 2781 \\
\hline Northern & 1 & $2(3)$ & 61.78 & 606 \\
\hline Southern & 2 & $2(2)$ & 43.11 & 1154 \\
\hline Yuki & 1 & $2(2)$ & 17.80 & 2500 \\
\hline
\end{tabular}

${ }^{a}$ A negative similarity score indicates that the words not referring to the same concept are more similar on average than words referring to the same concept, which means that the ASJP results do not bring support to this language group as a genealogical unit.

nearly average, producing roughly similar rates of lexical change as well. This situation is described by Brown (2006: 649-650), Ehret (2000:373), Jaxontov (1999:52), and Lohr (2000:219). The rate of lexical change ascertained by ASJP is perhaps best understood as expressing such an average.

The findings here can serve as a baseline for comparison with rates of change in other properties of languages, such as cognates (as in glottochronology), typological features, and also the size (number of languages) and geographical distribution of language groups. Properties found to have sufficiently uniform rates of change could be used to produce alternative dates or might be combined with ASJP lexical similarity for composite chronological estimation.

\section{Worldwide ASJP Chronology}

The same procedure described for the calibration groups can also be used to calculate ASJP dates for groups that lack alternative information about their time depth. Heggarty (2007) shows how even very rough estimates of linguistic time depth can be used in conjunction with archaeological information to infer sequences of historical events. Compared with the informal dates typically used for this purpose, ASJP dates have the advantages of a uniform definition and a quantitatively known level of accuracy.

Tables 3-7 present ASJP dates (years BP) for nearly all of the world's known language families, calculated by inserting the group average similarity into formula (3) where $s_{0}=$ $92 \%$ and $r=0.72$. The families are all defined in Ethnologue, sixteenth edition (Lewis 2009); the subgroups are augmented in three cases from the calibration sources as previously described. Also included are the highest-level subgroups of each family and, in some cases, groups of the next one or two lower taxonomic levels. The choice of which groups to include at lower levels is based on degree of attestation in the database, age (for older families we typically go further down into subgroups), and general interest. Languages in the ASJP database used to generate these dates include those currently spoken and also extinct languages attested by word lists collected from native speakers after $1700 \mathrm{CE}$.

The second column in tables 3-7 shows the number of pairs of lists involved in the calculations. Usually this number is smaller than the number of possible pairs formed from the languages in Ethnologue because some of the languages are not represented by lists in the ASJP database. Occasionally, 
Table 7. Automated Similarity Judgment Program (ASJP) dates for language groups of South America

\begin{tabular}{|c|c|c|c|c|}
\hline Group & Pairs & Subgroups & Similarity & Date \\
\hline Arauan & 17 & $4(5)$ & 28.88 & 1764 \\
\hline Arawakan & $\ldots$ & $\ldots$ & $\ldots$ & $\ldots$ \\
\hline Maipuran & 796 & $6(6)$ & 6.08 & 4134 \\
\hline Aymaran & 2 & $2(3)$ & 45.92 & 1057 \\
\hline Barbacoan & 8 & $3(4)$ & 12.16 & 3080 \\
\hline Cayapa-Colorado & 1 & $2(2)$ & 36.23 & 1419 \\
\hline Coconucan & 1 & $2(2)$ & 69.87 & 419 \\
\hline Cahuapanan & 1 & $2(2)$ & 42.23 & 1185 \\
\hline Carib & 72 & $2(2)$ & 19.50 & 2362 \\
\hline Northern & 38 & $5(5)$ & 19.37 & 2371 \\
\hline Southern & 11 & $3(3)$ & 18.74 & 2422 \\
\hline Chapacura-Wanham & 1 & $2(2)$ & 25.87 & 1931 \\
\hline Chibchan & 210 & $10(10)+1$ & 5.11 & 4400 \\
\hline Aruak & 6 & $3(3)+1$ & 14.61 & 2800 \\
\hline Guaymi & 2 & $2(2)$ & 10.62 & 3286 \\
\hline Kuna & 1 & $2(2)$ & 53.68 & 820 \\
\hline Rama & 1 & $2(2)$ & 3.19 & 5117 \\
\hline Talamanca & 9 & $4(4)$ & 15.30 & 2731 \\
\hline Choco & 7 & $2(2)$ & 20.87 & 2258 \\
\hline Embera & 12 & $2(2)$ & 51.76 & 875 \\
\hline Chon & 1 & $2(2)$ & 14.87 & 2774 \\
\hline Guahiban & 10 & $5(5)$ & 39.39 & 1291 \\
\hline Jivaroan & 6 & $4(4)$ & 58.94 & 678 \\
\hline Katukinan & 3 & $3(3)$ & 25.29 & 1965 \\
\hline Macro-Ge & 245 & $12(14)$ & .78 & 7266 \\
\hline Ge-Kaingang & 23 & $3(3)$ & 3.47 & 4989 \\
\hline Yabuti & 1 & $2(2)$ & 32.02 & 1607 \\
\hline Maku & 26 & $6(6)$ & 11.81 & 3124 \\
\hline Mascoian & 2 & $2(5)$ & 29.76 & 1718 \\
\hline Mataco-Guaicuru & 25 & $2(2)$ & 4.19 & 4701 \\
\hline Guaicuruan & 10 & $5(5)$ & 13.61 & 2909 \\
\hline Mataco & 10 & $5(7)$ & 18.96 & 2404 \\
\hline Nambiquaran & 3 & $3(3)$ & 14.55 & 2807 \\
\hline Panoan & 144 & $7(8)$ & 27.24 & 1853 \\
\hline North-Central & 5 & $3(6)$ & 22.64 & 2134 \\
\hline Northern & 3 & $3(5)$ & 44.70 & 1099 \\
\hline South-Central & 5 & $2(9)$ & 27.24 & 1853 \\
\hline Southeastern & 2 & $2(2)$ & 50.27 & 920 \\
\hline Quechuan & 18 & $2(2)$ & 29.77 & 1717 \\
\hline Quechua II & 17 & $2(3)$ & 48.51 & 974 \\
\hline Tacanan & 3 & $2(2)$ & 32.37 & 1590 \\
\hline Araona-Tacana & 2 & $2(2)$ & 40.04 & 1266 \\
\hline Tucanoan & 83 & $3(4)$ & 15.62 & 2699 \\
\hline Eastern Tucanoan & 30 & $2(3)$ & 40.70 & 1241 \\
\hline Western Tucanoan & 7 & $3(3)$ & 22.32 & 2156 \\
\hline Tupi & 570 & $8(10)$ & 8.73 & 3585 \\
\hline Monde & 9 & $4(5)$ & 29.87 & 1712 \\
\hline Munduruku & 1 & $2(2)$ & 34.79 & 1480 \\
\hline Tupari & 3 & $3(5)$ & 27.28 & 1850 \\
\hline Tupi-Guarani & 434 & $9(12)$ & 33.22 & 1550 \\
\hline Yuruna & 1 & $2(3)$ & 49.26 & 951 \\
\hline Uru-Chipaya & 2 & $2(2)$ & 33.89 & 1520 \\
\hline Witotoan & 12 & $2(2)$ & 2.49 & 5491 \\
\hline Boran & 2 & $2(2)$ & 20.69 & 2271 \\
\hline Witoto & 3 & $2(4)$ & 13.66 & 2903 \\
\hline Yanomam & 23 & $4(4)$ & 38.66 & 1319 \\
\hline Zamucoan & 2 & $2(2)$ & 14.96 & 2765 \\
\hline Zaparoan & 3 & $3(7)$ & 11.40 & 3178 \\
\hline
\end{tabular}

however, the number of pairs in column 2 is larger than the number of language pairs because some languages are represented by several lists for different dialects; this increase in sample size is expected to decrease the variance of the sampling distribution of similarity scores and to leave the mean unchanged.

The third column indicates the number of subgroups across which pairwise similarities are averaged to produce the similarity percentage. The parenthesized numbers in the third column indicate the total number of subgroups according to Ethnologue. This may be compared with the number of subgroups used in the calculations to get an idea of the completeness of the data from which a given date was estimated. For instance, in table 3, Afro-Asiatic has seven subgroups according to Ethnologue. A total of 24,303 pairs were drawn from six of these seven subgroups to produce the date of 6016 BP. When the number of subgroups used differs from the existing number of subgroups, there is the possibility for some eventual improvement in age estimates. The level of individual Ethnologue languages - that is, the level corresponding to a particular ISO-639-3 code-is treated as a taxonomic level in its own right. For instance, in table 5, the Arai (Left May) subgroup of Arai-Kwomtari is not further subclassified in Ethnologue, so the four languages belonging to this subgroup are each treated as coordinate branches under the Arai (Left May) node.

The similarities include a few languages that are missing from Ethnologue (being recently extinct or newly described) but that are represented in the ASJP database and assigned to subgroups according to the classifications in the sources for the lists. The number of such languages (or their subgroups) is indicated following a plus sign after the number of Ethnologue subgroups. For instance, in table 3, the ASJP dates for Yeniseian are based on two languages included in Ethnologue plus four extinct languages in two extinct subgroups. Finally, the groups named in tables 3-7 for which no information exists are those that are presented in Ethnologue as including only one subgroup or for which the ASJP database includes languages for only one subgroup.

The last two columns show the similarity score and the ASJP date. Three characteristics of the dates are worth noting. First, some of the families reported in Ethnologue are controversial and may not be phylogenetically real. If subgroups of a family are not in fact genetically related but instead similar only because of contact and diffusion, the lexical similarity score is expected to be relatively small and the date not meaningful. Second, the Ethnologue classification of many groups is conservative in the sense that it does not include subgroups identified in other classifications. If the classification of a group is too conservative and fails to introduce subgrouping where it should, the average similarity score for the group will be inflated and the time depth will be underestimated. Third, a few higher-order groups are estimated to be younger than some of their immediate subgroups. These anomalies may reflect random variation in the similarities, mistakes in 
the classification, or unusually high rates of lexical change in the apparently older subgroups.

Tables 3-7 represent the first attempt known to us to assign dates to most if not all of the world's language families using a uniform method and database. These dates are based on the Ethnologue classification because of its comprehensiveness and availability, and Ethnologue names of groups are used for consistency. Although tables 3-7 are restricted to the top few levels of the Ethnologue classification, dates for all Ethnologue groups represented in the ASJP database are available as a $\mathrm{PDF}$ in $\mathrm{CA}+$ online supplement A. It may also be useful to generate ASJP dates based on other classifications identified by specialists as more accurate than those of Ethnologue. To facilitate the calculation of such dates, our database and software have been made available online (Wichmann et al. $2010 b$ and Holman 2010, respectively).

\section{Acknowledgments}

We would like to thank the scholars who commented on earlier versions of this work: Gene Anderson, Peter Bellwood, Roger Blench, Robert Blust, W. South Coblin, Michael Coe, Bernard Comrie, Mark Donohue, Chris Ehret, Michael Fortescue, Anthony Grant, Russell Gray, Terrence Kaufman, Victor H. Mair, Johanna Nichols, Andrew Pawley, Robert Rankin, Malcolm Ross, Laurent Sagart, Paul Sidwell, Brian Stross, Edward Vajda, and James Watters. Our gratitude also extends to five anonymous reviewers.

\section{Comments}

\section{Willem F. H. Adelaar}

Leiden University Centre for Linguistics, P.O. Box 9515, 2300 RA Leiden, The Netherlands (w.f.h.adelaar@hum .leidenuniv.nl). 8 VI 11

The Automated Similarity Judgment Program (ASJP) offers an alternative for the classic glottochronology method and presents distinct advantages in terms of objectivity and accuracy. Its outcome, however, remains the product of an automated process, which cannot replace the "protracted linguistic analysis required for cognate identification" (as claimed in this article by Holman et al.). Historical-comparative linguists will always feel the necessity to reproduce the identification of cognates in a nonquantitative manner in order to see for themselves whether the languages in any specific language pair are significantly similar and how they are similar. The ASJP can help them to make the selection of language pairs to be analyzed first.

The ASJP chronology represented in tables 3-7 provides an overview of the time depths of most of the world's linguistic families. Some dates are remarkable. For instance, the time depths given for Mayan (2,220) and Mixe-Zoquean $(1,407)$ are smaller than those commonly assumed by linguists working with these languages. The time depth given for MixeZoquean is incompatible with the circumstance that a variety of Zoquean intermediate between Proto-Zoquean (ASJP: AD 593) and Proto-Mixe-Zoquean (ASJP: AD 213) has been associated with the Epi-Olmec inscriptions of the Mesoamerican Late Preclassic (400 BC-AD 200; cf. Justeson and Broadwell 2007:412).

By contrast, Oregon Penutian, computed at 11,886 years, is surpassed in time depth only by Khoisan. Its antiquity is also reported to be more than double that of Penutian as a whole. It follows that the calculation must be in error or that part of Oregon Penutian is not Penutian but constitutes a separate family.

There are other cases in which the ASJP provides a greater time depth for a lower node in a tree than for its higher pendent (e.g., Tangut-Qiang, Eastern Malayan-Polynesian, and Rama). Furthermore, an exaggerated antiquity is attributed to Insular Celtic $(3,876)$ and to Ge-Kaingang $(4,989)$.

For some of the language families listed, the absence of important phylogenetic branches arouses curiosity. A lack of living descendants may underlie the absence of the Egyptian branch of Afro-Asiatic, but that Korean is not mentioned, either as an Altaic branch or independently, is surprising.

The calibration exercise in which ASJP data are contrasted with published dates defining the moment of divergence of language families on the basis of archaeological, historical, or epigraphic evidence is not felicitous. The selection of language groups is arbitrary, several areas are underrepresented, and it contains cases of overlapping (e.g., Dardic, Indo-Aryan, IndoIranian, and Iranian). The proposed dates for some of the language groups (Saami, Mississippi Valley Siouan, etc.) are partly or entirely based on linguistic criteria, although the supporting evidence has been classified as archaeological.

The weakness of this procedure is that it seeks to exclude information obtained by historical-comparative linguistic research. Apparently, in a search for objectivity, calibration sources, selected in an uncritical way, have been restricted to disciplines in which language data play no role. Thus, the outcomes of well-established nonquantitative linguistic research are willfully ignored. The examples of Sorbian and Tupi-Guaraní can illustrate this point.

Sorbian is attributed a divergence date around 1550, when "Lower and Upper Lusatian developed from the more or less homogeneous Lusatian in the 16th century, i.e., at the time of the reformation" (Fodor 1962:132). This statement is in conflict with the majority of linguistic research concerning these well-studied languages. In the sixteenth century, the two Sorbian languages were linguistically and geographically distinct (although their status as separate languages may not have been an issue of contention at that time). It has been assumed that the two languages originated from two separate WestSlavic migrations (cf. Stone 1972). During the late Middle 
Ages, the two communities were separated by sparsely inhabited marshlands that were gradually drained from the sixteenth century on, which resulted in increased language contact and the rise or consolidation of transitional dialects. There was probably never such thing as a "more or less homogeneous Lusatian." Note that the lexical similarity between the two Sorbian languages $(68.78 \%)$ is hardly greater than that attributed to Czech-Slovak (67.18\%) with an assumed divergence date of AD 950.

For Tupi-Guaraní, the article refers to archaeological and ethnohistoric information suggesting that "the ancestors of the Guarani and the ancestors of the Tupinambá evolved independently since 2000-1500 BP." It should be observed that Tupi-Guaraní is an extensive language family comprising scores of languages dispersed over much of South America, so the exclusive focus on Guaraní and Tupinambá seems arbitrary. The article ignores all the linguistic reconstruction work that has been done for Tupi-Guaraní (Dietrich 1990; Jensen 1998; Rodrigues 1984-1985, etc.). It also misses the provocative suggestion that "the break-up of Proto-TupiGuaraní may have occurred as late as the 13th century AD" (Schleicher 1998:326) based on the observation that sixteenthcentury Tupinambá was nearly identical to a reconstructed Tupi-Guaraní protolanguage.

In order to establish the added value of the ASJP, it would be advisable to confront its outcomes with the best achievements of nonquantitative linguistic reconstruction, not with opinions and guesses derived from disciplines that are not necessarily congenial with a linguistic way of thinking.

\section{Peter Bellwood}

School of Archaeology and Anthropology, Australian National University, Canberra, Australian Capital Territory 0200, Australia (peter.bellwood@anu.edu.au). 17 VI 11

Any new dating technique that can illuminate the chronology of past human dispersals is potentially of great importance. The litmus test, of course, is whether the dates are "correct." Glottochronology using the Swadesh formula has had a checkered history because of the recognition that different languages have had different rates of lexical diversification that depended on their linguistic environments. Blust (2000), in a critique of lexicostatistics and glottochronology, has already discussed this problem for Austronesian languages. Because I am neither a linguist nor a statistician, I cannot pronounce directly on the merits or demerits of this new method that uses Levenshtein distances, and I am unsure whether or how it tackles this problem of different rates of lexical change.

Instead, as an archaeologist and a prehistorian of ancient migration, I can comment best on three points. First, what does a protolanguage tell us about human history? If the language family concerned was always confined roughly within the region where it exists today, then the answer may be "very little." Thus, the date of 14,592 years for the first recorded divergence within the Khoisan languages probably has no connection whatsoever with any significant migration or cultural invention. More likely, it just reflects the vagaries of survival-those Khoisan languages that happen to exist today just happen to coalesce around this date, which might well be close to some hypothetical limit of the method in terms of the extent to which it can count back in time.

However, the majority of the protolanguages considered belong to very large language families that spread very widely in prehistory. An ancestral Indo-Aryan language was introduced into South Asia, just as an ancestral Polynesian one was introduced into Polynesia. Given the complete lack of any convincing linguistic evidence to suggest that Proto-IndoEuropean was spoken in India or Proto-Austronesian in Tahiti, the histories of these language families inevitably involve major questions of human migration. But the dating of any protolanguage will not mark the actual colonization of a new region. Every protolanguage had a prelanguage within which innovations developed that would in due course be shared uniquely by the spreading daughter languages within the ensuing subgroup. A protolanguage must therefore be later in time than the initial spread of its common ancestral prelanguage. We can get a hint of prelanguage time depth from the Romance languages; Proto-Romance is calibrated by these authors to 1,729 years ago (AD 282), but the spread of Latin (Pre-Romance) during the Roman Empire to conquered provinces outside Italy took place mainly between $150 \mathrm{BC}$ and $\mathrm{AD} 100$. So the time lag here averaged about 300 years. This suggests that to work from any protolanguage back to the original population dispersal that gave rise to it, we might need to add around 300 years (or more?) to the dates presented.

This brings up the second point. How good are the calibrations? For those labeled as archaeological and thus prehistoric, a minority seem to me to be rather questionable, but those that relate to colonizations of previously uninhabited regions are probably close to being correct. The best examples here are the first arrivals of humans on specific islands within the Austronesian speaking world. The calibrated dates given for Malayo-Polynesian (MP; 4250 BP), Eastern Malayo-Polynesian (EMP; $3350 \mathrm{BP}$ ), and East Polynesian colonization (but not protolanguage breakup) are acceptable because the islands concerned (Batanes, Island Melanesia beyond the Solomons, and Eastern Polynesia) were uninhabited before Austronesian arrival. The Automated Similarity Judgment Program (ASJP) date for Eastern Polynesia seems to be good in this regard, at $7 \%$ less than the calibration, hence in accord with the above discussion. But MP is $41 \%$ too young, and EMP is $13 \%$ too old. I am puzzled as to why, between the calibrated dates and the ASJP dates, there should be so much variation that seems to swing randomly from plus to minus by factors of up to $100 \%$. Perhaps the authors can comment on this in their 
response. Are we back with issues concerning varying rates of lexical change through time?

A third and final point concerns language-family completeness. Protolanguages can be reconstructed only if daughter languages survived long enough to be recorded. IndoEuropean is considered in this paper without Anatolian or Tocharian, so the calculations presumably do not relate to true Proto-Indo-European (or Indo-Hittite) but to a lesser entity. Families such as Indo-European and Sino-Tibetan have seen enormous expansions of some of their subgroups in historical times, swamping and eradicating unrecorded smaller cousins. Tai, Malayic, Chamic, Mon-Khmer, Vietnamese, Indo-Aryan, and Tibeto-Burman languages have all done this to a deeper layer of Austro-Asiatic "tribal" languages that probably once existed throughout Mainland Southeast Asia and northeastern India. Many language families are remnant, and we cannot be sure that their earlier history will ever be recorded clearly by comparing modern spoken languages alone. This is not a criticism, just a fact of life that all linguistic prehistorians must face.

\section{Robert Blust}

Department of Linguistics, University of Hawai'i, Manoa, 569 Moore Hall, 1890 East-West Road, Honolulu, Hawaii 96822, U.S.A. (blust@hawaii.edu).6 V 11

Lexicostatistics and its offshoot glottochronology were inspired by two primary desiderata: (1) the desire for a "quick and easy" method to subgroup languages that does not require knowledge of phonological history and (2) the hope that this method could provide absolute dates for linguistic splits. As a general program intended to expand the tool kit of historical linguistics, these goals are admirable and legitimate. The problem with their implementation has always been the difficulty of providing a guarantee of trustworthiness.

In creating the Automated Similarity Judgment Program (ASJP), Holman et al. have altered the glottochronological formula $t=\log C /(2 \log r)$ by replacing "cognate percentage" $(C)$ with a "similarity score" $(s)$ based on Levenshtein distance (LD) and then modifying $s\left(\log s-\log s_{0}\right)$ to reflect the not unreasonable assumption that protolanguages probably were dialectally complex. Apart from this they have inherited the flawed conceptual machinery of the past, most crucially what Blust (2000) called the universal constant hypothesis (UCH), namely, the claim by Lees (1953) that $90 \%$ of randomly sampled languages will show a retention rate between $78.7 \%$ and $82.3 \% /$ millennium on the Swadesh 200-item list. They reference this critique but appear to dismiss it on the grounds that the data are drawn from a single family. However, as noted by Blust (2000), such a dismissal implies that language families vary in retention rate-an assumption that is equally fatal to the UCH. In fact, Holman et al. conclude that there are "no differences among families in the rate or variability of lexical change." If this is true and the best-studied case (Austronesian, with 224 data points) fails to support the $\mathrm{UCH}$, one would expect greater caution: if lexicostatistics/glottochronology is unreliable for Austronesian, then it and its ASJP variant are unreliable in general. Given this problem, it is surprising that the authors do not incorporate a rate-smoothing algorithm as used by scholars working with Bayesian inference (e.g., Atkinson and Gray 2005; Greenhill and Gray 2009) or quantify the error in the date estimates to render the true range of their figures more transparent.

The substitution of $s$ for $C$ clearly is intended to solve a problem that plagued lexicostatistics, where cognate decisions made by nonspecialists were often based on "inspection" (English translation: guesswork). This change is understandable in a project of global scope, because even a team of 15 coauthors cannot be expected to know enough about the historical phonology of the world's languages to distinguish cognation from convergence in more than a few families. But the use of LD introduces other complications. In comparing languages with little sound change, LD may well match cognate identification in most cases. However, where sound change has been extensive, the use of LD creates a problem analogous to that of variation in retention rate. Consider the North Sarawak branch of Austronesian, which has been called a "hot spot" for sound change (Blust 2007). Bintulu ba, Kiput dufih

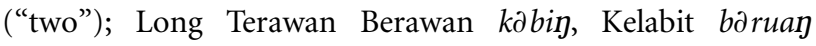

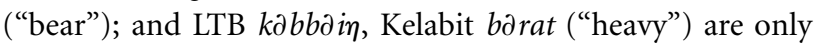
a few of many nonobvious cognates. Regardless of how LD is calculated for cases such as these, it must be higher than that for many noncognates (e.g., Bintulu $a i$ : Kiput $a k \partial m$ ["leg/foot"], Bintulu musus : Kiput masa ["rub"], etc., which require fewer "editing changes" to achieve a match). The upshot of this approach is that degree of sound change translates into separation time, and variation in rate of lexical replacement is then compounded with variation in rate of phonological change, leading to a double layer of distortion.

Apart from these fundamental considerations affecting the theory on which the conclusions of this study are based, the exposition could have benefited from the use of data to illustrate some of the more critical assumptions, as in discussing the difference between LD and normalized LD. The expository use of data may also have clarified some contradictions in table 3, as where Niger-Congo is dated at 6227 BP but the Atlantic-Congo subgroup is dated earlier at 6525, as well as similar cases with several other families. In some cases an appeal to "objectivity" by using Ethnologue phylogenies seems pointless, as in recognizing a "Plains" branch of Algonkian (cf. Goddard 1996:4-5, where nothing of the kind exists) or an "Altaic" language family, which has virtually no defenders.

Finally, Holman et al. are to be congratulated on making a serious effort to determine the reliability of ASJP dates by checking them against historical documents, epigraphy, and archaeological inference. However, this is not always consis- 
tent, as where the surprisingly shallow date for Austronesian conflicts with the (unmentioned) evidence for the presence in Taiwan two millennia earlier of Neolithic cultures that must have been ancestral to the modern aborigines (Tsang 2005). Moreover, because by their own account the mean absolute discrepancy between ASJP dates and those obtained through more secure methods is $29 \%$, with five off by more than $50 \%$, it is hard to see how the authors of this study can expect many linguists to embrace this attempted revival of a faulty approach to linguistic chronology: "new clothes" or not, the emperor is still parading in the raw.

\section{Koen Bostoen}

Royal Museum for Central Africa, Universiteit Gent, Université Libre de Bruxelles, Leuvensesteenweg 13, 3080 Tervuren, Belgium (koen.bostoen@africamuseum.be).6 V 11

This paper comes up with a computerized and purportedly superior alternative to glottochronology. Its authors clearly belong to those scholars who are "still attached to the idea that lexicostatistics and glottochronology can be fixed, that if only we can get the technical aspects rights, the results will be of value" (Blench 2006:41). They admit, though, that glottochronology "has had a checkered history." This is rather euphemistic. Many historical linguists would categorically reject the historical significance of any quantitative study relying on such a limited and purely lexical data set, especially if it is used not only to produce a preliminary genealogical classification of languages but also to calculate absolute time elapsed since languages diverged. Such an unconditional position is motivated by reasons of all sorts that cannot be reiterated here. The most fundamental one is no doubt that languages do not necessarily change at a constant rate, not even their basic lexicon, which is assumed to be more resistant to borrowing. All depends on the "ecology of language evolution" (Mufwene 2001). This is also the main reason why I have always been rather pessimistic about the possibility of a universally valid formula for calculating the rate of language change. The Automated Similarity Judgment Program (ASJP) does not move away from the original Swadesh hypothesis but rather tries to make its testing more sophisticated.

This article adds to a trend that has risen for a decade or so: a renewed interest in quantitative lexical approaches to language classification. In my own field of study, several recent studies (Holden 2002; Holden and Gray 2006; Holden, Meade, and Pagel 2005; Rexová, Bastin, and Frynta 2006) have applied new phylogenetic methods to the Bastin, Coupez, and Mann (1999) data set, the largest lexicostatistical Bantu study. These new approaches all share the following features: (1) they rely on the cognacy judgments of Bastin, Coupez, and Mann (1999), (2) their involvement of linguists is inexistent or minimal, and (3) they apply statistical procedures whose results most linguists have to take for granted. The ASJP study is superior to those in that it shares only the last feature with them. It did involve historical linguists who considered certain intricacies of language change. Moreover, it did not rely on preestablished cognate percentages but applied a new method of calculating lexical similarity on a newly collected data set. Despite these improvements, this study still raises many doubts and questions, such as the following.

The ASJP works with 40 of the Swadesh 100-item list. Five of these supposedly most stable items were previously defined more precisely ("skin") or omitted entirely from the Bantu list because of cultural inappropriateness ("hand") or rather grammar than lexis (I, we, you; Bastin, Coupez, and Mann 1999).

The ASJP method determines lexical similarity on the basis of Levenshtein (edit) distances instead of cognate percentages. This method has so far been applied mainly in dialectology. When lexical variation is studied at the microscale, sound change is a significant dialectometric parameter. However, because Levenshtein measurement of similarity treats all changes as equivalent without regard to their phonological plausibility or historical frequency, it is questionable whether this method can simply be extrapolated to more distantly or nonrelated languages on a worldwide scale. At the macrolevel, to exclude convergent phonological shifts, only typologically rare sound changes or chain shifts are historically relevant. Moreover, crucial phonological information is lost because of their standardized orthography; especially, ignoring features such as tone and vowel length reduces the validity of their results.

The ASJP applies a uniform analytical approach to a single database of worldwide coverage. Automated similarity judgment is presumed more objective than expert cognate identification. Nevertheless, morphological information seems to be ignored. Morphological change does not necessarily run parallel with phonological and lexical change. In order to avoid comparing apples and oranges, the measurement of lexical similarity should be more sensitive to morphology.

The ASJP aspires to outdo glottochronology in computing the absolute time elapsed since languages diverged. The classical formula is adapted and checked against 52 calibration points instead of the traditional 13 points. Their coverage is also geographically wider and no longer limited to languages with a long written tradition of almost exclusively IndoEuropean origin. Calibration is based on published historical, epigraphic, and archaeological dates. This should be seen as an improvement. I remain very hesitant, though, about the validity of both the calibration dates and the obtained time depths, certainly when considering the data I am most familiar with. The Benue-Congo calibration date of 6,500 years seems to rest on a partial misinterpretation of a tentative association we made between certain phenomena observed in the archaeological record of the Cameroonian Grassfields and the introduction of Benue-Congo languages in that particular re- 
gion and not the emergence of a distinct Benue-Congo branch itself that must be older (Bostoen and Grégoire 2007). Likewise, the calculated time depths for Niger-Congo and BenueCongo - that is, $6227 \mathrm{BP}$ and $4940 \mathrm{BP}$, respectively-are significantly shallower than experts tend to assume. The time depth generally associated with Bantu is 5000 BP, while NigerCongo is estimated at $12,000-10,000$ BP. All this does not rouse optimism about the possibility of a universally valid formula for calculating the rate of language change.

\section{Lyle Campbell}

Department of Linguistics, University of Hawai'i, Manoa, 569 Moore Hall, 1890 East-West Road, Honolulu, Hawaii 96822, U.S.A. (lylecamp@hawaii.edu). 8 V 11

Vocabulary changes for many reasons-social, cultural, cognitive, and others. There is nothing in how words change that suggests that anything constant or lawlike should be expected. Scholars will doubt the claim of a "constant rate of decrease for lexical similarity" and the dates from the Automated Similarity Judgment Program (ASJP).

Holman et al.'s computerized alternative to glottochronology for estimating time elapsed since parent languages diverged into daughter languages is based on the purportedly 40 most stable items from the Swadesh 100-word list. They determine automated judgments of lexical similarity from Levenshtein distance (LD). Real cognates are not required, because complicating effects from borrowing and from comparison of unrelated languages are mentioned.

$\mathrm{LD}$ is defined as the minimum number of successive changes necessary to convert one word into another. How "changes" involving ASJP orthographic symbols are counted for LD raises questions. Why is the distance between Spanish weso and Italian osso ("bone") 3 (for Spanish to Italian: $1 \mathrm{~s}$ added, $2 w$ deleted, $3 e$ to $o$ )? Many see Spanish we as a diphthong, a single unit, with we to $o$ as one change, not two changes, with a distance for the word pair of 2 , not 3 . If a substitution occurs multiply in a word, does it count as 1 or several? For example, does the $p$ to $b$ correspondence in Lithuanian pāpas/Middle High German buoben ("breast") count as 2 separate substitutions or 1 , a single change of $p$ to $b$ that simultaneously affects all occurrences of the same sound in a word? The $p: b$ "correspondence" violates expectations of Grimm's law-the forms involve onomatopoeia or nursery formation, illustrating one problem with counting similarities in this way. Different decisions about how to count changes skew the results.

About claimed stability, from the 40-word list, person, mountain, and skin are loans in English; words for name, sun, and star are loanwords in numerous languages; and words for breast (as above) and dog are similar across numerous languages because of onomatopoeia (breast reflecting nursing or sucking sounds). It might be claimed that statistically such examples average out; however, with calibration points from only five languages of the Americas (from ca. 180 of the world's ca. 350 families), is confidence warranted in the determination of stability for such items?

There is subjectivity in the choices of calibration points. The authors recognize that "there are various difficulties in identifying languages with archaeological materials," saying, "we nevertheless use archaeological calibrations because they are the only ones available for chronologically deep families." For example, they date Benue-Congo at 6500 based on arrival of macrolithic tools and pottery, and they correlate Mississippi Valley Siouan with squash cultivation and Tupi-Guarani with ceramics and other archaeological materials. These are hardly satisfying, because technological innovation and agriculture often diffuse across linguistic boundaries. The interpretation of epigraphic information for a split between Eastern and Western Cholan is far from uncontroversial. Are calibration points of this sort from only 17 language families sufficient to warrant confidence in the method and its dates?

The phylogenetic classification follows Ethnologue (Lewis 2009); however, Ethnologue's classification is notoriously flawed-Holman et al. say, "some of the families reported in Ethnologue are controversial and may not be phylogenetically real." The following from Ethnologue that figure in the paper are rejected or highly controversial: Altaic, Andamanese, Gulf (rejected even by Mary Haas, who proposed it), Hokan, Khoisan (now mostly considered an areal grouping), Mataco-Guaicuruan, Na-Dene, Nilo-Saharan, Oregon Penutian, Penutian, and Witototan (Boran is not Witotoan, though it shows much diffusion). Panoan and Tacanan are given as separate families but are one, Pano-Tacanan. The authors suggest that restricting attention to Ethnologue's classification, though faulty, "avoids the subjectivity entailed in making choices between competing classifications that could be biased toward a particular result." However, Ethnologue's abundant known errors cannot give better results than consensus classifications for individual families that are more accurate than Ethnologue's.

The difference between calibration dates and ASJP-calculated dates range from -90 to +119 , with $29 \%$ mean absolute discrepancy. In several cases, "higher-order groups are estimated to be younger than some of their immediate subgroups" (assumed daughters), for example, Pama-Nyungan 4295 but Paman 4918; Austronesian 3633 but Eastern MalayoPolynesian (branch of Austronesian) 3803; Algonquian 3343 but Plains (branch of Algonquian) 5002; "Hokan" 4915 but "Northern Hokan" 5666 and Karok-Shasta (branch of Northern Hokan) 5246; "Penutian" 5522 but "Oregon Penutian" a whopping 11,886; and Chibchan 4400 but Rama (a branch of Chibchan) 5117. With so much variation in the results, how are we to trust the results for particular cases? The ASJP dates differ widely from those of both standard glottochronology and Russell Gray's Language and Culture Evolution Group (http://language.psy.auckland.ac.nz/ 
publications), though less accurate in many cases. For reasons such as these, I believe scholars will find it difficult to accept the ASJP and its dates.

\section{Sheila Embleton}

Department of Languages, Literatures and Linguistics, York University, South 561 Ross Building, 4700 Keele Street, Toronto, Ontario M3J 1P3, Canada (embleton@yorku.ca). 9 V 11

This is a very interesting paper that opens up promising new avenues for further investigation by linguists as well as others investigating linguistic prehistory such as archeologists and anthropologists. It is not that the ideas are new or revolutionary so much as that this method relies on large-scale computing power of a type not yet dreamed of in the early days of Swadesh's investigations into this topic in the 1950s or even the later versions a generation after in the 1980s (Embleton 1986 for a summary). But it is this type of paper that reliably moves our discipline forward. I would urge further research — whether by this team or others-on how well this method works in situations where there truly is either more rapid change or large amounts of borrowing - the "constructed languages, creoles, mixed languages, and pidgins" mentioned in the first paragraph of supplement A. Although I appreciate the reason for restricting the database to 40 words in each language, I think this is shortsighted for a number of reasons, and it gives some "short-term gain for long-term pain." We often do not know where the methods and databases will next take us, and thus it is incumbent on us all, wherever possible, to construct databases that are maximally robust for future usage for multiple purposes and by multiple diverse users who may have different goals. Given the ubiquity of the Swadesh 100-meaning list (even with slight variations), I would urge that the database be augmented-again, whether by this team or others is immaterial-to at least a 100-meaning version if not a 200 -meaning version. This would make the database useful for others doing totally unrelated research. Despite the fact that a few researchers, such as Holman et al. (2008), have found that a subset of 40 more stable items can yield results comparable with those from a 100-meaning Swadesh list, I believe that most linguists will remain unconvinced of the results of mathematical or statistical methods with such limited data per language treated to (what they will see as) an overly simplistic similarity measure (just looking at substitution, deletion, and insertion rather than the naturalness/"phonological plausibility" or frequency of certain types of substitutions, deletions, or insertions; ignoring tone, suprasegmentals, vowel length) as opposed to, for example, the more nuanced type of distance measure used already many years ago by Grimes and Agard (1959). Given my background and own research, I am as aware as anybody of the fact that simplifying assumptions often prove remarkably valid, often surprisingly so. Earlier they were more necessary or justified because of the limits of computing power, although this is no longer the case. However, advances in such methods will be of limited use if we cannot persuade others not only to use them but also to feel confident in their results. Additionally, I have noted that in much recent work, the Ethnologue (Lewis 2009) is cited as a kind of benchmark or reference point on classification. I do not quarrel with that at all, but in the spirit of robust scientific inquiry, the groupings and subgroupings used there should now be scrutinized carefully by linguists from a wide variety of backgrounds. Thus, I see this paper as a major step forward, and I call for more research both around all aspects of the underpinnings of this method (scrutiny of the Ethnologue, collection of more word lists, collection of longer word lists, more nuanced similarity/dissimilarity measures) and taking this method forward into less charted territory. I hope that this method can provide a useful heuristic, perhaps providing some quick yet accurate results, but that it also does not discourage the eventual painstaking work of full reconstruction, examination of cognate matches, and so on, as per the more traditional methods.

\section{Johanna Nichols}

Department of Slavic Languages and Literatures, University of California, Mailcode 2979, 6303 Dwinelle, Berkeley, California 94720-2979, U.S.A. (johanna@berkeley.edu). 10 VI 11

This automated method is much needed and offers a potentially major improvement to the historical linguist's tool kit. I see three areas in which some further honing is needed.

1. Calibration. Though the calibration language families are well chosen and well researched, some of their assumed ages require fine-tuning. The separation of Czech and Slovak isoglosses began before the tenth century, but the languages remained in close contact after that, doubtless exchanging lexemes and thus foreshortening any calculated age. The historically determined separation of East Slavic is only one factor in the linguistic distance between Russian and Ukrainian; Ukrainian is a plain East Slavic language, while Russian descends from a diglossic fusion of East Slavic and the South Slavic roots of medieval Church Slavic (Uspenskij 2002). This makes East Slavic unsuitable for calibration despite its accurate historical datability. The archaeological date of 4,400 years for Indo-Iranian is probably too old; Anthony (2007: 371-411) identifies the Sintashka culture (ca. 2100-1800 BCE) as ancestral Indo-Iranian. (The Poltavka culture, identified with Indo-Iranian by Holman et al., is one of two cultures probably ancestral to Sintashta, and it lacks the essential IndoIranian cultural attributes first found in Sintashta.) Similarly, the archaeological date for Indo-Aryan should probably be Anthony's 1800-1600 BCE for the southern frontier of the Petrovka culture (Anthony 2007:454). All of these observa- 
tions bring the benchmark dates closer to the Automated Similarity Judgment Program (ASJP)-calculated dates and suggest that the technique is on the whole more accurate than the authors find.

2. Test data. The Ethnologue (Lewis 2009) classification is easy to use but in many cases does not reflect current knowledge of subgrouping or family status. Holman et al. predict that "if subgroups of a [putative] family are not in fact genetically [i.e., genealogically, phylogenetically] related but instead similar only because of contact and diffusion, the lexical similarity score is expected to be relatively small and the date not meaningful." However, the test data include several nonfamilies that nonetheless give ordinary-looking scores: NigerCongo (some of whose branches have not in fact been shown to be related; Güldemann 2010, 2011), Altaic (for its status see Janhunen 1996, 2001; Schönig 2003), North Caucasian, Australian, and Gulf. Each of these has been or is thought to be a family by some linguists, but no demonstration of relatedness has been given. I am not sure that the linguistic evidence for the Trans-New Guinea group is probative, but the argument that the earliest horticulture in the New Guinea Highlands should have resulted in a large language spread across the highlands is strong (e.g., Pawley 2006; Ross 2005). However, that argument indicates a dispersal date closer to 9,000 years, while the ASJP dates it at 6,609. Penutian is a debated group that may in fact meet the statistical criteria for familyhood (Nichols 2010:369-370) but that is evidently too old to preserve detectable regular correspondences. It must have originated in the lacustrine environment of the postglacial eastern Cascades and Sierra Nevada and moved westward as this environment desiccated; on archaeological evidence its Miwok-Costanoan subbranch reached California about 5,000 years ago. Further high-level subgrouping makes Penutian considerably older than that. Somewhat as for Trans-New Guinea, Penutian could be a family only if it is much older than the ASJP age of 5,522 years. All of these groups should have returned identifiably anomalous dates but do not do so.

Among well-established families, ASJP dates for older families appear to be systematically too young. Afro-Asiatic must be much older than the ASJP 6,016 years. Its Egyptian daughter branch is attested from ca. $5400 \mathrm{BP}$ and is grammatically and lexically quite distant from its sisters, suggesting that much more than 616 years passed between the Afro-Asiatic dispersal and the first attestation of Egyptian. The ASJP dates the Semitic branch of Afro-Asiatic at 3,301 years, but at ca. 4,500 years ago, Akkadian and Eblaite were distinct within East Semitic, and Amorite was still more distinct and NonEast Semitic, so Semitic must be no less than about 5,000 years old. (In fact this epigraphic dating would make Semitic another good calibrating family.)

The ASJP dates Uralic at 3178, but its daughter branch Proto-Finno-Ugric came into contact with Proto-IndoIranian and early Iranian, plausibly represented by the Sintashta culture (Anthony 2007:385; Koivulehto 2001), ca. 4000 BP. Its other daughter branch Samoyedic may have contacted
Proto-Tocharian somewhat earlier (Janhunen 1983), and Proto-Uralic and Proto-Indo-European have possible lexical contacts (papers in Carpelan, Parpola, and Koskikallio 2001; overview in Janhunen 2009). Thus, Uralic is of at least IndoEuropean-like antiquity.

3. Interpretation. Inadequately provided for is the situation where daughter languages separate phonologically but remain in contact, foreshortening lexically based dates. Embleton (1986, 1991) tackles this situation for Germanic and Romance; Slavic is another case. An automated technique needs to be able to handle or at least identify this not uncommon situation.

\section{Colin Renfrew}

McDonald Institute for Archaeological Research, University of Cambridge, Downing Street, Cambridge CB2 3ER, United Kingdom (acr10@cam.ac.uk). 18 IV 11

It is certainly interesting that glottochronology seems to be gaining a new lease on life and encouraging to see contradicted the aphorism that "linguists don't do dates" (McMahon and McMahon 2006). I was initially impressed by the output of estimated dates for more than 500 of the world's language families. Impressive also the use of a code that can be produced with any QWERTY keyboard, thus avoiding any phonetic considerations whatever, and the somewhat Procrustean decision to reduce the 100-item Swadesh list to 40 items.

My suspicions, however, were first aroused, albeit at a rather superficial level, by the striking contrast with the results obtained in the rather different approach of Gray, Atkinson, and Greenhill (2011). How could they come to such different conclusions, particularly for the Indo-European family? And why, with the single exception of the age assigned to the Khoisan family (14.5 kyr BP), were all the resultant ages here so young?

My initial response is that for ages of more than 2,000 years, the dates proposed may indeed be consistently too young. There are perhaps two main reasons for that. First, the set of 52 calibration dates has several oddities both of inclusion and of omission. But second, the regression line in figure 2 has the obvious feature that all the points for ages greater than $3500 \mathrm{BP}$ lie above the regression line rather than spread across it, while most of the dates younger than 2000 BP lie well below the line. This may suggest that a linear regression is not the best way to express the observed variability and therefore that the Swadesh formula may need further refinement.

A related question is the avoidance, in the calibration data set, of well-documented and well-dated ancient languages such as Maya, Aztec, Mycenaean Greek, Hittite, and the epigraphically recorded languages of the Near East, including Ancient Egyptian. Surely, many of the words on the 40-item list are known in these languages. And if language pairs are 
desired, should we not be comparing Tocharian and Hittite and indeed Mycenaean-all well-documented Indo-European languages?

Perhaps most serious of all, however, is the inclusion in the "calibration" list of languages that are in fact dated on the basis of specific historical hypotheses that themselves contain hidden chronological assumptions. Such are the estimates here for Dardic, Indo-Aryan, Indo-Iranian, and Iranian offered by Parpola (1999) and for Indo-European by Anthony (2007). All these pertain to a debatable view of Indo-European origins: alternative assumptions could lead to much earlier dates. If one calls the date for Benue-Congo (6500 BP) into question as dubious and notes that the family status of PamaNyungan is debated, the three remaining calibration points for languages with assigned dates before $3500 \mathrm{BP}$ are all located in Malayo-Polynesia. The exercise then loses geographical generality.

The overall initiative that underlies this paper is warmly to be welcomed, but these critical observations are more than mere quibbles. The difficulty in obtaining secure data points with ages greater than 2,000 years should encourage the more systematic use of the few languages for which reliable records survive from before that time. It is possible that evidence would then emerge to justify the initial reaction that many of these 52 age calibrations are too young and that this conclusion might therefore be carried forward to most of the resulting estimates offered here.

\section{Paul Sidwell}

Department of Linguistics, Languages, and Cultures, Australian National University, LPO Box 8139, Canberra 0200, Australia (paulsidwell@yahoo.com). 17 VI 11

This paper emerges from the Automated Similarity Judgment Program (ASJP) based at the Department of Linguistics, Max Planck Institute for Evolutionary Anthropology (Leipzig). Readers should know that I am a member of the ASJP consortium and that in September 2010 I accepted travel to Leipzig paid by the institute to attend their First Conference on ASJP and Language Prehistory (ALP-I).

The paper reports on progress with a new approach to calculating the time depth of language splits that avoids some of the pitfalls of the traditional glottochronological method. First of all, there is no identification of cognates; instead, that is an objective automatic computation of phonetic similarity. Second, measures of similarity are compared with known calibration points across a number of language families, so rates of change may be more representative than the typically narrower empirical studies that have gone before.

However, I do have a number of concerns. The meat and potatoes of the project is the comparison of lists of 40 words from each language retranscribed into the ASJPcode, which reduces the list of possible phonetic contrasts to a modest fixed universal list. Thus, it is clear that what are being compared are tiny subsets of linguistic entities that are structurally simplified compared with their source forms, and these subsets are then asked to stand as useful representative samples of their respective namesakes. These simplified words are compared, segment by segment, and similarity is calculated on the presence or absence of one-to-one agreement between segments. The comparisons are made "without regard to their phonological plausibility or historical frequency." For example, a pair such as /tap, tak/ is as similar as /tap, tip/ because each differs by just one substitution.

Trials conducted with longer lists or with algorithms that incorporated measures of phonological plausibility are said to have provided no better results (Holman et al. 2008). Remarkably, this was interpreted by the investigators as a positive, because they could proceed with the much less burdensome work (manually and computationally) of using more complete lexical and/or phonological data. This is of real concern to me. With this "no worse than" approach, it strikes me that the project may be setting the bar so low that it is inherently difficult to assess any of its results.

Also, why are the data being manually retranscribed into ASJPcode? None of the offered justifications is valid in the era of Unicode, when even a simple numeric keyboard is sufficient to enter any International Phonetic Alphabet symbol. It is a computationally trivial task to read fully phonemic data and automatically merge distinctions at the discretion of the programmer without affecting the original database. This would have internal and external advantages: (1) the project could manipulate the reading of the data to empirically test the results at various levels of representation and (2) this publicly funded data set would be useful to other projects that may wish to run experiments on a large comparative set of real data. It is lamentable that the project did not anticipate these.

The attempt to calibrate language splits dated by external means is certainly commendable, and it is an approach already seen recently in related projects using somewhat different methods (e.g., Gray and Atkinson 2003; Greenhill, Drummond, and Gray 2010). Yet the actual results obtained so far by the ASJP are fairly disappointing. The paper reports finding that "strong correlation supports the critical claim that log lexical similarity decreases linearly as time depth increases." That is reasonably trivial because we would not have expected much else. The really critical result, however, is how well the spread of data points converges or diverges to this function. In this case the empirical findings are that "the mean absolute percent discrepancy is $29 \%$; of the 52 ASJP dates, five are off by more than $50 \%$, and one is off by more than $100 \%$." This is pretty poor indeed; in practice, offered an ASJP date of, say, $2000 \mathrm{BC}$ for your language split, you really do not know in which millennium it took place. Again, the bar is set at "no worse than" traditional glottochronology, and it is not clear to me just what has been gained.

In conclusion, it is clear that this is a project that has a 
long way to go before it may begin to pay real dividends. Real attempts to introduce objective measures into comparative linguistics deserve serious support, but please, let us do so with an underlying set of real data that can be widely accessed and manipulated under the widest reasonable range of parameters. This is the only way we can really test when it is really doing something useful or when it is failing to do so.

\section{George Starostin}

Center for Comparative Linguistics, Russian State University for the Humanities, 125993 Miusskaya Place, 6 Moscow, Russia (gstarst@rinet.ru). 3 V 11

Writing from the "traditional" standpoint of a historical-comparative linguist, I do not feel sufficiently qualified to evaluate the technical details of the algorithm proposed by the authors (although I suspect that it could not be significantly improved on without expanding the linguistic basis of the comparison). Instead, I would prefer to directly address its results, asking such questions as would seem to be the most important for a historical linguist. (a) Do these results yield important new information? (b) Do they confirm, in a new way, any of our previously voiced hypotheses? (c) Can the procedure be considered useful enough to be replicated in the future on additional data, most importantly, that of poorly studied language families?

Question $a$ must be answered in the positive. It may now be considered proven that in some cases automated use of the Levenshtein distance method to measure lexical similarity yields chronological results that are close to traditional results of comparing historical linguistic information with extralinguistic sources of chronological information ("calibrated" dates). Unfortunately, in other cases it does not, and it cannot be reasonably well predicted which particular cases will work and which ones will not. Even though the mean absolute discrepancy in the results, as stated by the authors, is $29 \%$ (not a critical figure, per se), historical linguists are on the whole more interested in specific histories of lineages than averaged statistics, and such transparently erroneous Automated Similarity Judgment Program (ASJP) dates as shown by Indo-Aryan, Turkic, and Mongolic results, among others (I list only the undebatable cases), are not likely to cause much excitement among specialists.

Hence, a negative answer to the second question $b$ : the results achieved neither confirm nor disprove any of the previous dating attempts. This, in turn, strengthens skepticism about the idea that relative and absolute chronology can be safely established based on automatic measurement of phonetic similarity. On average, it seems reasonable to assume that different degrees of phonetic dissimilarity between related languages may translate to different time depths of separation, but reality teaches us that this is far from always the case. Notable exceptions happen both on the "microscale" - as can be observed on the published ASJP world tree, where numerous languages are classified unsatisfactorily within small branches-and the "macroscale"-for instance, when the phonological system of one descendant of a former macrofamily remains archaic whereas that of its other descendant undergoes a "breakdown" through convergence with a nonrelated family.

The ASJP's greatest advantage is objectivity. But when objectivity is understood as crude automatization that sacrifices most of the achievements of the comparative method (including not only biased assumptions but also rigorous conclusions) in favor of testing out a scenario that has proved to be wrong in more than one case, the only benefits from such an objective approach are purely statistical and will not be of much use to linguists working on particular families.

The answer to the final question $c$ is, based on these considerations, self-evident: although the ASJP's approach is innovative and may occasionally, even at the current stage of development, yield results that could trigger productive ideas on the part of historical linguists, its efficiency will at best have "compulsive" rather than "convincing" force, that is, stimulating linguists into exploring certain paths through indirect statistical hints rather than concrete evidence. Such stimulation per se is not at all a bad thing, but I cannot help wondering just how many linguists that endorse glottochronology would want to explore chronological hypotheses based on the ASJP's statistics rather than the regular application of the original formulas, such as the original Swadesh method or the improved procedure in Starostin (2000)—or, for that matter, how many linguists skeptical of glottochronology will become less skeptical of it upon reading the paper.

That said, as a member of the "proglottochronology" camp, I side completely with the authors on one extremely positive aspect of the paper, namely, the confirmation, through their calibration method (occasional quibbles about the archaeological dates notwithstanding), of the reality of a regular (on average) rate of lexical change, which may further encourage the much too often misunderstood and unjustly dismissed glottochronological studies. It shows that "manual" lexicostatistics, in which selection of cognates is often hampered by insufficient knowledge about the historical phonology and areal connections of the languages, need not be afraid of occasional mistakes if even fully automated lexicostatistics, armed with 40 -item lists and a complete lack of historical information, confirms the general validity of the method. To that end, it would be interesting at some point to combine the ASJP method with the complex manual procedure of Swadesh list evaluation currently employed in the Global Lexicostatistical Database (Starostin 2010), because it is my firm belief that automatic and manual procedures of historic analysis of linguistic material should complement each other rather than vie for the same space. 


\section{Reply}

Comments indicate areas for improvement in data, methods, and results, for which we are grateful. With the online availability of the Automated Similarity Judgment Program (ASJP) database and software, readers are provided with tools to implement for themselves suggested improvements and any other possible enhancements. Below, in response to comments, we assess prospects for progress in automated dating of language families.

\section{Data}

Calibrations. Adelaar, Bostoen, Campbell, Nichols, and Renfrew question 14 of the 52 calibration points. For the remaining 38 points, the average percent discrepancy between calibration and ASJP dates in table 1 is $25 \%$ compared with $29 \%$ for all 52 points. This is consistent with Nichols's suggestion that the $29 \%$ figure is conservative if anything. Improvements in the calibrations will not come easily. As an indication of the difficulties, commentators actually suggest only two alternative calibration dates: Nichols cites younger dates for both Indo-Iranian and Indo-Aryan, but Renfrew offers the opposing view that calibration dates for these two groups should be older.

Classifications. Blust, Campbell, Embleton, Nichols, and Renfrew question the adequacy of Ethnologue (Lewis 2009) as a source for language classification. Classification errors relating to calibration dates are especially critical because calibrations are the basis for estimating all other dates. However, only one of the 52 calibration points is challenged because of its classification. Renfrew notes that "the family status of Pama-Nyungan is debated," although strong evidence in favor of the group has been presented by Alpher (2004), O'Grady and Hale (2004), and Harvey (2009). With this one exception, groups based on questioned classifications are not relevant to constructing the formula for calculating ASJP dates presented in tables 3-7. More felicitous classifications for these and any other groups can be used to estimate alternative ASJP dates with the aid of our publicly accessible data and software.

Ancient languages. Bellwood, Nichols, and Renfrew mention long-extinct languages preserved in textual materials. The ASJP database currently includes word lists from 48 such languages. Most of these lists are problematic for dating because they appear to be compiled from an assortment of materials with little or no indication of their respective exact ages or even whether the extracted words are all contemporary. Lists based on ancient textual materials are probably more useful in research on topics other than dating languagegroup divergence.

Forty-item lists. Bostoen, Campbell, Renfrew, and Sidwell mention the small number of referential items of ASJP lists, and Embleton explicitly recommends expanding lists to 100 or even 200 items. Experiments varying the sizes of word lists have been undertaken since Holman et al. (2008), where 40 items were found to be just as adequate for classificatory purposes as 100 items. Most recently, Kurniati et al. (2011) report minimal differences in the results of classifying a set of 20 Malay variants using the standard 40 ASJP items and lists with as many as 1,542 items. Longer lists may nevertheless be preferable for some purposes. Expanded lists are compatible with the organization of the ASJP database, which currently contains several hundred 100-item lists.

ASJPcode. Bostoen, Embleton, Renfrew, and Sidwell comment on the simplified coding system for sounds. Lexical data come in different types of phonological and orthographic conventions, depending on their sources. ASJPcode was developed to unify and simplify these representations into a system that requires no more phonological differentiation than is available in most of the sources. For instance, sources often underdifferentiate distinctions such as vowel length or tone or use non-International Phonetic Alphabet vowel symbols whose exact phonetic values cannot be determined. Using fine-grained transcriptions for some languages while being required to use coarse-grained ones for others would introduce inconsistency in the measurement of lexical distances among languages.

\section{Methods}

Measuring lexical similarity. Blust, Bostoen, Campbell, Embleton, and Sidwell comment on the similarity measure based on Levenshtein distance (LD). The first two ASJP papers (Brown et al. 2008; Holman et al. 2008) used a different measure based on the number of items that satisfied a set of context-based rules for matching. Alternative sets of matching rules were tried, but no improvement was achieved until these were replaced with LD (Bakker et al. 2009; Wichmann and Holman 2009). This replacement produced noticeably higher agreement with expert classifications. Since then, measures assigning different weights to some of the changes that are weighted equally in LD have been tried with no additional improvement observed. Other researchers are now testing different string similarity algorithms using the ASJP database. Huff and Lonsdale (2011) find no appreciable difference between the performance of the normalized LD divided (LDND) measure preferred by ASJP and that of ALINE (Kondrak 2000), the latter of which is sensitive to the phonetic features underlying phonemes. Pompei, Loreto, and Tria (2011) include tests of LDND against LDN and find that the former has a superior performance.

Rate constancy. Our formula for estimating dates from similarities employs a single rate of lexical change. Blust, Bostoen, and Campbell doubt the plausibility of a constant rate and the usefulness of the formula. Our calibrations confront these doubts with objective evidence on the margin of error expected for dates produced by the formula. Blust specifically mentions his study (Blust 2000) that presents data inconsis- 
tent with rate constancy in glottochronology. Like all of glottochronology, Blust's test relies on judgments of cognacy. His test also involves human judgment at other levels because it compares percentages of cognates shared by different modern languages with their reconstructed hypothetical ancestral language. Thus, greater differences in cognate percentages than expected from rate constancy could imply variation in rates of lexical change, variation in judgments of cognacy, or variation introduced by the process of language reconstruction. These sorts of alternative explanation are excluded by automated methods not dependent on human decisions. The ASJP database is therefore ideal for testing hypotheses about rate constancy and evaluating possible factors that might produce variation in rates. One step in that direction is our finding of no significant differences among language families in rate of lexical change; another is the observation by Wichmann and Holman (2009) that number of speakers of a language has little or no influence on its rate of change.

\section{Results}

Groups younger than subgroups. If the breakup of a language group is closely followed chronologically by the breakup of an immediate subgroup, the known variability of ASJP dates may influence the subgroup to show an ASJP date older than that for the larger group to which it belongs. Adelaar, Blust, and Campbell mention a total of 11 such cases, which have an average discrepancy equal to $26 \%$ of the younger date, similar to the $29 \%$ average calibration error. While such discrepancies might be viewed as awkward results for ASJP chronology, they are nonetheless anticipated within the margin of error of the approach.

Unexpected dates. Adelaar, Bellwood, Blust, Bostoen, Nichols, and Starostin mention ASJP dates inconsistent with received wisdom. Many of these discrepancies are not extreme compared with the margin of error for ASJP dates. Discrepancies for ASJP dates older than about 5000 BP cannot be evaluated in terms of calibrations, all of which involve ASJP dates younger than $5000 \mathrm{BP}$. Therefore, the relatively few cases of extreme discrepancies involving ASJP dates younger than $5000 \mathrm{BP}$ are those most likely either to be successfully challenged by commonly assumed dates or to replace those dates.

Usefulness of ASJP chronology. Blust, Sidwell, and Starostin suggest that the margin of error for ASJP dates is too great for the dates to be useful. Nevertheless, the margin of error is narrow enough sometimes to exclude competing proposed dates based on other methods. For example, Kaufman (1976) calculates a glottochronological breakup date of 4200 BP for Proto-Mayan, a date that has become accepted wisdom, especially by Mesoamerican archaeologists (e.g., Sharer and Traxler 2006). This is $89 \%$ older than the ASJP date of 2220 $\mathrm{BP}$, a discrepancy exceeded by only three of the 52 calibrations. Awareness of this incompatibility might lead to new and interesting insights into early Maya prehistory. In another instructive example, this one involving preglottochronological thinking in a postglottochronological age, Whistler (1983) declares a 5,000-year time depth for Proto-Pomoan based on an arbitrary-looking archaeological equation. In contrast, ASJP chronology yields a date of $1277 \mathrm{BP}$, meaning that Whistler's date differs from ours by $292 \%$. This discrepancy is much greater than found for any of the calibrations, permitting the safe rejection of Whistler's date.

—Eric W. Holman, Cecil H. Brown, Søren Wichmann, André Müller, Viveka Velupillai, Harald Hammarström, Sebastian Sauppe, Hagen Jung, Dik Bakker, Pamela Brown, Oleg Belyaev, Matthias Urban, Robert Mailhammer, Johann-Mattis List, and Dmitry Egorov

\section{References Cited}

Adelaar, Alexander. 2005. Malayo-Sumbawan. Oceanic Linguistics 44: 357-388.

- 2006. The Indonesian migrations to Madagascar: making sense of the multidisciplinary evidence. In Austronesian diaspora and the ethnogenesis of people in Indonesian archipelago: proceedings of the international symposium. T. Simanjuntak, I. H. E. Pojoh, and M. Hisyam, eds. Pp. 205-232. Jakarta: Indonesian Institute of Sciences, LIPI.

Aikio, Ante. 2006. On Germanic-Saami contacts and Saami prehistory. Suomalais-Ugrilaisen Seuran Aikakauskirja: Journal de la Société Finno-Ougrienne 91:9-55.

Alpher, Barry. 2004. Pama-Nyungan: phonological reconstruction and status as a phylo-genetic group. In Australian languages: classification and the comparative method. Claire Bowern and Harold Koch, eds. Pp. 93-126. Amsterdam: Benjamins.

Anthony, David W. 1995. Horse, wagon and chariot: Indo-European languages and archaeology. Antiquity 65:554-565.

- 2007. The horse, the wheel, and language: how Bronze Age riders from the Eurasian Steppes shaped the modern world. Princeton, NJ: Princeton University Press. [JN]

Atkinson, Quentin D., and Russell D. Gray. 2005. Are accurate dates an intractable problem for historical linguistics? In Mapping our ancestry: phylogenetic methods in anthropology and prehistory. C. Lipo, M. O’Brien, S. Shennan, and M. Collard, eds. Pp. 269-296. Chicago: Aldine. $[\mathrm{RB}]$

Bakker, Dik, André Müller, Viveka Velupillai, Søren Wichmann, Cecil H. Brown, Pamela Brown, Dmitry Egorov, Robert Mailhammer, Anthony Grant, and Eric W. Holman. 2009. Adding typology to lexicostatistics: a combined approach to language classification. Linguistic Typology 13:167-179.

Bastin, Y., A. Coupez, and M. Mann. 1999. Continuity and divergence in the Bantu languages: perspectives from a lexicostatistic study. Annales, Sciences Humaines, vol. 162. Tervuren: Royal Museum for Central Africa. $[\mathrm{KB}]$

Bellwood, Peter. 2007. Southeast China and the prehistory of the Austronesians. In Lost maritime cultures: China and the Pacific. Tianlong Jiao, ed. Pp. 36-53. Honolulu: Bishop Museum.

Bellwood, Peter, and Peter Hiscock. 2005. Australia and the Austronesians. In The human past: world prehistory and the development of human societies. Chris Scarre, ed. Pp. 264-305. London: Thames \& Hudson.

Bergsland, Knut, and Hans Vogt. 1962. On the validity of glottochronology. Current Anthropology 3:115-153.

Blench, R. 2006. Archaeology, language and the African past. Lanham, MD: Altamira. $[\mathrm{KB}]$

Blust, Robert. 2000. Why lexicostatistics doesn't work: the “universal 
constant" hypothesis and the Austronesian languages. In Time depth in historical linguistics, vol. 2. Colin Renfrew, April McMahon, and Larry Trask, eds. Pp. 311-331. Cambridge: McDonald Institute for Archaeological Research.

2007. Òma Lóngh historical phonology. Oceanic Linquistics 46:1-53. [RB]

Bostoen, Koen, and Claire Grégoire. 2007. La question bantoue: bilan et perspectives. Mémoires de la Société de Linguistique de Paris 15: 73-91.

Bremmer, Rolf H., Jr. 2009. An introduction to Old Frisian: history, grammar, reader, glossary. Amsterdam: Benjamins.

Brochado, José Joachim Justiniano Proenza. 1984. An ecological model of the spread of pottery and agriculture into eastern South America. PhD dissertation, University of Illinois, Urbana-Champaign.

Brown, Cecil H. 2006. Glottochronology and the chronology of maize in the Americas. In Histories of maize. John Staller, Robert Tykot, and Bruce Benz, eds. Pp. 647-663. Amsterdam: Elsevier.

Brown, Cecil H., Eric W. Holman, Søren Wichmann, and Viveka Vilupillai. 2008. Automated classification of the world's languages: a description of the method and preliminary results. STUF: Language Typology and Universals 61:285-308.

Bury, J. B. 1923. A history of the later Roman Empire from the death of Theodosius I to the death of Justinian (A.D. 395 to A.D. 565). London: Macmillan.

Carpelan, Christian, Asko Parpola, and Petteri Koskikallio, eds. 2001. Early contacts between Uralic and Indo-European: linguistic and archaeological considerations. Suomalais-Ugrilaisen Seuran Toimituksia, 242. Helsinki: Suomalais-Ugrilainen Seura. [JN]

Castillo, Dennis. 2006. The Maltese cross: a strategic history of Malta. Westport, CT: Praeger Security International.

Dahl, Otto Christian. 1951. Malgache et Maanjan: une comparaison linguistique. Oslo: Egede-Instituttet.

Dietrich, Wolf. 1990. More evidence for an internal classification of Tupi-Guarani languages. Berlin: Mann. [WFHA]

Dixon, R. M. W. 1997. The rise and fall of languages. Cambridge: Cambridge University Press.

Ehret, Christopher. 2000. Testing the expectations of glottochronology against the correlations of language and archaeology in Africa. In Time depth in historical linguistics, vol. 1. Colin Renfrew, April McMahon, and Larry Trask, eds. Pp. 373-399. Cambridge: McDonald Institute for Archaeological Research.

Embleton, Sheila M. 1986. Statistics in historical linguistics. Bochum: Brockmeyer.

- 1991. Mathematical methods of genetic classification. In Sprung from some common source: explorations into the prehistory of languages. S. M. Lamb and E. D. Mitchell, eds. Pp. 365-388. Stanford, CA: Stanford University Press. [JN]

Evans, Nicholas, and Rhys Jones. 1997. The cradle of the PamaNyungans: archaeological and linguistic speculations. In Aboriginal Australia in global perspective: archaeology and linguistics. Patrick McConvell and Nicholas Evans, eds. Pp. 385-417. Melbourne: Oxford University Press.

Fodor, Istvan. 1962. Comment on "On the validity of glottochronology." Current Anthropology 3:131-134.

Fortescue, Michael D. 1998. Language relations across Bering Strait: reappraising the archaeological and linguistic evidence. London: Cassell.

Goddard, Ives. 1996. Introduction. In Languages, vol. 17 of Handbook of North American Indians. Ives Goddard, ed. Pp. 1-16. Washington, DC: Smithsonian Institution. [RB]

Golden, Peter B. 1998. The Turkic peoples: a historical sketch. In The Turkic languages. Lars Johanson and Éva Á. Csató, eds. Pp. 16-29. London: Routledge.

Gray, R. D., Q. D. Atkinson, and S. J. Greenhill. 2011. Language evolution and human history: what a difference a date makes.
Philosophical Transactions of the Royal Society B 366:1090-1100. [CR]

Gray, Russell D., and Quentin D. Atkinson. 2003. Language-tree divergence times support the Anatolian theory of Indo-European origin. Nature 426:435-439. [PS]

Green, Roger C. 2003. The Lapita horizon and traditions: signature for one set of Oceanic migrations. In Pacific archaeology: assessments and prospects. Christophe Sand, ed. Pp. 95-120. Nouméa: Service des Musées et du Patrimonie de Nouvelle-Calédonie.

Greenhill, S. J., R. Blust, and R. D. Gray. 2008. The Austronesian basic vocabulary database: from bioinformatics to lexomics. Evolutionary Bioinformatics 4:271-283.

Greenhill, Simon J., Alexei J. Drummond, and Russell D. Gray. 2010. How accurate and robust are the phylogenetic estimates of Austronesian language relationships? PLoS One 5(3):e9573. [PS]

Greenhill, Simon J., and Russell D. Gray. 2009. Austronesian language phylogenies: myths and misconceptions about Bayesian computational methods. In Austronesian historical linguistics and culture history: a festschrift for Robert Blust. Alexander Adelaar and Andrew Pawley, eds. Pp. 375-397. Canberra: Pacific Linguistics. [RB]

Grimes, Joseph E., and Frederick B. Agard. 1959. Linguistic divergence in Romance. Lanquage 35:598-604.

Güldemann, Tom. 2010. Sprachraum and geography: linguistic macroareas in Africa. In Language mapping, vol. 2 of Language and space: international handbook of language variation. Alfred Lameli, Roland Kehrein, and Stefan Rabanus, eds. Pp. 561-585. Berlin: Mouton de Gruyter. [JN]

- 2011. Proto-Bantu and Proto-Niger-Congo: macroareal typology and linguistic reconstruction. In Geographical typology and linguistic areas, with special reference to Africa. Osamu Hieda, Christa König, and Hirosi Nakagawa, eds. Pp. 109-141. Amsterdam: Benjamins. [JN]

- Forthcoming. Changing profile when encroaching on hunter-gatherer territory: towards a history of the Khoe-Kwadi family in Southern Africa. In Hunter-gatherers and linguistic history: a global perspective. Tom Güldemann, Patrick McConvell, and Richard Rhodes, eds. Cambridge: Cambridge University Press.

Hammarström, Harald. 2010. A full-scale test of the language farming dispersal hypothesis. Diachronica 27:197-213.

Harvey, Mark. 2009. The genetic status of Garrwan. Australian Journal of Linguistics 29:195-244.

Haugen, Einar. 1982. Scandinavian language structures: a comparative historical survey. Minneapolis: University of Minnesota Press.

Heeringa, Wilbert, Peter Kleiweg, Charlotte Gooskens, and John Nerbonne. 2006. Evaluation of string distance algorithms for dialectology. In Linguistic distances. J. Nerbonne and E. Hinrichs, eds. Pp. 51-62. Workshop at the joint conference of International Committee on Computational Linguistics and the Association for Computational Linguistics, Sydney. http://urd.let.rug.nl/nerbonne/ papers/heeringa-et-al-coling-2006.pdf.

Heggarty, Paul. 2007. Linguistics for archaeologists: principles, methods and the case of the Incas. Cambridge Archaeological Journal 17:311-340.

Holden, C. J. 2002. Bantu language trees reflect the spread of farming across sub-Saharan Africa: a maximum-parsimony analysis. Proceedings of the Roval Societv B 269:793-799. [KB]

Holden, C. J., and R. D. Gray. 2006. Rapid radiation, borrowing and dialect continua in the Bantu languages. In Phylogenetic methods and the prehistory of languages. P. Forster and C. Renfrew, eds. Pp. 19-31. Cambridge: McDonald Institute for Archaeological Research. $[\mathrm{KB}]$

Holden, C. J., A. Meade, and M. Pagel. 2005. Comparison of maximum parsimony and Bayesian Bantu language trees. In The evolution of cultural diversity: a phylogenetic approach. R. Mace, C. J. Holden, and S. Shennan, eds. Pp. 53-65. London: UCL. [KB]

Holman, Eric W. 2010. Program for calculating ASJP dates (ver. 1.0). 
http://email.eva.mpg.de/ wichmann/software.htm (accessed August 2010).

Holman, Eric W., Søren Wichmann, Cecil H. Brown, Viveka Velupillai, André Müller, Pamela Brown, and Dik Bakker. 2008. Explorations in automated language classification. Folia Linguistica 42:331-354.

Huff, Paul, and Deryle Lonsdale. 2011. Positing language relationships using ALINE. Lanquage Dynamics and Change 1:128-162.

Humphreys, Humphrey Lloyd. 1993. The Breton language: its present position and historical background. In The Celtic languages. Martin J. Ball and James Fife, eds. Pp. 606-643. London: Routledge.

Hymes, Dell H. 1960. Lexicostatistics so far. Current Anthropology 1: 3-44.

Jackson, Kenneth. 1951. Common Gaelic: the evolution of the Goedelic languages. Proceedings of the British Academy 37:71-97.

Janhunen, Juha. 1983. On early Indo-European-Samoyed contacts. In Symposium saeculare Societatis Fenno-Ugricae. Juha Janhunen, ed. Pp. 115-128. Helsinki: Finno-Ugric Society. [JN]

1996. Prolegomena to a comparative analysis of Mongolic and Tungusic. In Proceedings of the 38th Permanent International Altaistic Conference. Giovanni Stary, ed. Pp. 209-218. Wiesbaden: Harrasowitz. [JN]

2001. Indo-Uralic and Ural-Altaic: on the diachronic implications of areal typology. In Early contacts between Uralic and Indo-European: linguistic and archaeological considerations. Christian Carpelan, Asko Parpola, and Petteri Koskikallio, eds. Pp. 207220. Helsinki: Suomalais-Ugrilainen Seura. [JN]

2003. Proto-Mongolic. In The Mongolic languages. Juha Janhunen, ed. Pp. 1-29. London: Routledge.

- 2009. Proto-Uralic: what, where, and when? In The quasquicentennial of the Finno-Ugrian Society. Juha Janhunen, ed. Pp. 57-78. Helsinki: Suomalais-Ugrilainen Seura. [JN]

Jaxontov, S. 1999. Glottochronology: difficulties and perspectives. In Historical linguistics and lexicostatistics. V. Shevoroshkin and P. J. Sidwell, eds. Pp. 51-59. Melbourne: Association for the History of Language.

Jensen, Cheryl. 1998. Comparative Tupí-Guaraní morphosyntax. In Handbook of Amazonian languages, vol. 4. Desmond C. Derbyshire and Geoffrey K. Pullum, eds. Pp. 489-618. Berlin: Mouton de Gruyter. [WFHA]

Justeson, John S., and George A. Broadwell. 2007. Language and languages of Mesoamerica. In The legacy of Mesoamerica: history and culture of a Native American civilization, 2nd edition. Robert M. Carmack, Janine L. Gasco, and Gary H. Gossen, eds. Pp. 407438. Upper Saddle River, NJ: Pearson Prentice-Hall. [WFHA]

Kaufman, Terrence. 1976. Archaeological and linguistic correlations in Mayaland and associated areas of Meso-America. World Archaeology 8:101-118.

Kessler, Brett. 1995. Computational dialectology in Irish Gaelic. In Proceedings of the seventh conference of the European Chapter of the Association for Computational Linguistics. Pp. 60-66. San Francisco: Kaufmann.

Koivulehto, Jorma. 2001. The earliest contacts between Indo-European and Uralic speakers in the light of lexical loans. In Early contacts between Uralic and Indo-European: linguistic and archaeological considerations. Christian Carpelan, Asko Parpola, and Petteri Koskikallio, eds. Pp. 235-263. Helsinki: Suomalais-Ugrilainen Seura. [JN]

Kondrak, Grzegorz. 2000. A new algorithm for the alignment of phonetic sequences. Proceedings of the first meeting of the North American Chapter of the Association for Computational Linguistics. Pp. 288-295. N.p.: Association for Computational Linguistics.

Kropp-Dakubu, Mary Esther, ed. 1977-1980. West African language data sheets. Legon, Ghana: West African Linguistic Society.

Kurniati, Santi, Yessy Prima Putri, Søren Wichmann, and David Gil.
2011. Tapan: an exploration in Malayic subgrouping. Paper presented at ISMIL 15, Universitas Islam Negri Malang, June 24-26.

Lees, Robert B. 1953. The basis of glottochronology. Lanquage 29: 113-127.

Lewis, M. Paul, ed. 2009. Ethnologue. 16th edition. Dallas: SIL International. http://www.ethnologue.com.

Lohr, M. 2000. New approaches to lexicostatistics and glottochronology. In Time depth in historical linguistics, vol. 1. Colin Renfrew, April McMahon, and Larry Trask, eds. Pp. 209-222. Cambridge: McDonald Institute for Archaeological Research.

Matras, Yaron. 2002. Romani: a linguistic introduction. Cambridge: Cambridge University Press.

McMahon, A., and R. McMahon. 2006. Why linguists don't do dates: evidence from Indo-European and Australian languages. In Phylogenetic methods and the prehistory of languages. P. Forster and C. Renfrew, eds. Pp. 153-160. Cambridge: McDonald Institute for Archaeological Research. [CR]

Mitchell, Donald. 1990. Prehistory of the coasts of southern British Columbia and northern Washington. In Handbook of North American Indians, vol. 7. Wayne Suttles, ed. Pp. 340-358. Washington, DC: Smithsonian National Museum of Natural History.

Moraes Farias, P. F. de. 2003. Arabic medieval inscriptions from the Republic of Mali: epigraphy, chronicles, and Songhay-Tuarreg history. Oxford: Oxford University Press.

Mufwene, S. S. 2001. The ecology of language evolution. Cambridge Approaches to Language Contact. Cambridge, New York: Cambridge University Press. [KB]

Nichols, Johanna. 2010. Language families, macroareas, and contact. In The handbook of language contact. Raymond Hickey, ed. Pp. 361-379. London: Blackwell. [JN]

Nichols, Johanna, and Tandy Warnow. 2008. Tutorial on computational linguistic phylogeny. Lanquage and Linquistics Compass 2: 760-820.

Norman, Jerry. 1988. Chinese. Cambridge: Cambridge University Press.

O'Grady, Geoff, and Ken Hale. 2004. The coherence and distinctiveness of the Pama-Nyungan language family within the Australian linguistic phylum. In Australian languages: classification and the comparative method. Claire Bowern and Harold Koch, eds. Pp. 69-92. Amsterdam: Benjamins.

Parpola, Asko. 1999. The formation of the Aryan branch of IndoEuropean. In Artefacts, languages and texts, vol. 3 of Archaeology and language. Roger Blench and Matthew Spriggs, eds. Pp. 180207. London: Routledge.

Pawley, Andrew K. 2006. Trans New Guinea languages. In Encyclopedia of language and linguistics. Keith Brown, ed. Pp. 17-21. Amsterdam: Elsevier. [JN]

- 2009. The role of the Solomon Islands in the first settlement of Remote Oceania: bringing linguistic evidence to an archaeological debate. In Austronesian historical linguistics and culture history: a festschrift for Robert Blust. Alexander Adelaar and Andrew Pawley, eds. Pp. 515-540. Canberra: Pacific Linguistics.

Pohl, Walter. 2004. Die Germanen, vol. 57 of Enzyklopädie deutscher Geschichte. Munich: Oldenbourg.

Pompei, Simone, Vittorio Loreto, and Francesca Tria. 2011. On the accuracy of language trees. PLoS ONE 6(6):e20109, doi:10.1371/ journal.pone.0020109.

Pugh, Stefan M. 2007. Introduction and phonology, vol. 1 of A new historical grammar of the East Slavic languages. Munich: LINCOM Europa.

Ramsay, S. Robert. 1987. The languages of China. Princeton, NJ: Princeton University Press.

Rankin, Robert L. 2006. Siouan tribal contacts and dispersions evidenced in the terminology for maize and other cultigens. In Histories of maize. John Staller, Robert Tykot, and Bruce Benz, eds. Pp. 563-575. Amsterdam: Elsevier. 
Renfrew, Colin, April McMahon, and Larry Trask, eds. 2000. Time depth in historical linguistics. Cambridge: McDonald Institute for Archaeological Research.

Rexová, K., Y. Bastin, and D. Frynta. 2006. Cladistic analysis of Bantu languages: a new tree based on combined lexical and grammatical data. Naturwissenschaften 93:189-194. [KB]

Rodrigues, Aryon Dall'Igna. 1984-1985. Relações internas na familia lingüística Tupí-Guaraní. Revista de Antropologia 27/28:33-53. [WFHA]

Róna-Tas, A. 1991. An introduction to Turkology. Szeged, Hungary: Universitas Szegediensis de Attila József.

Ross, Malcolm, and Åshild Næss. 2007. An Oceanic origin for Äiwoo, the language of the Reef Islands? Oceanic Linquistics 46:456-498.

Ross, Malcolm D. 1988. Proto Oceanic and the Austronesian languages of western Melanesia. Canberra: Pacific Linguistics.

- 2005. Pronouns as a preliminary diagnostic for grouping Papuan languages. In Papuan pasts: investigations into the cultural, linguistic, and biological history of the Papuan-speaking peoples. Andrew K. Pawley, Robert Attenborough, Robin Hide, and Jack Golson, eds. Pp. 15-66. Canberra: Research School of Pacific and Asian Studies, Australian National University. [JN]

Sagart, Laurent. 1999. The roots of Old Chinese. Amsterdam: Benjamins.

Sagart, Laurent, Roger Blench, and Alicia Sanchez-Mazas. 2005. Introduction. In The peopling of East Asia: putting together archaeology, linguistics and genetics. Laurent Sagart, Roger Blench, and Alicia Sanchez-Mazas, eds. Pp. 1-14. London: RoutledgeCurzon.

Sapir, Edward. 1916. Time perspective in aboriginal American culture: a study in method. Geological Survey of Canada, Memoir 90, Anthropological Series, no. 13. Ottawa: Government Printing Bureau.

Schenker, Alexander M. 1995. The dawn of Slavic: an introduction to Slavic philology. New Haven, CT: Yale University Press.

Schleicher, Charles Owen. 1998. Comparative and internal reconstruction of the Tupi-Guarani language family. $\mathrm{PhD}$ dissertation, University of Wisconsin, Madison. [WFHA]

Schönig, Claus. 2003. Turko-Mongolic relations. In The Mongolic languages. Juha Janhunen, ed. Pp. 403-419. London: Routledge. [JN]

Séguy, Jean. 1971. La relation entre la distance spatiale et la distance lexicale. Revue de Linguistique Romane 35:335-357.

Serva, Maurizio, and Filippo Petroni. 2008. Indo-European languages tree by Levenshtein distance. Europhysics Letters 81(6):68005, doi: 10.1209/0295-5075/81/68005.

Sharer, Robert J., and Loa P. Traxler. 2006. The ancient Maya. 6th edition. Stanford, CA: Stanford University Press.

Sidwell, Paul. 2006. Dating the separation of Acehnese and Chamic by etymological analysis of the Aceh-Chamic lexicon. Mon-Khmer Studies 36:187-206.

Starostin, George. 2010. Preliminary lexicostatistics as a basis for language classification: a new approach. Journal of Language Relationship 3:79-117. [GS]

Starostin, Sergei. 2000. Comparative linguistics and lexicostatistics. In Time depth in historical linguistics, vol. 1. Colin Renfrew, April McMahon, and Larry Trask, eds. Pp. 223-265. Cambridge: McDonald Institute for Archaeological Research. [GS]

Stone, Gerald. 1972. The smallest Slavonic nation: the Sorbs of Lusatia. London: Athlone Press of the University of London. [WFHA]

Swadesh, Morris. 1950. Salish internal relationships. International Journal of American Linquistics 16:157-167.

- 1955. Towards greater accuracy in lexicostatistic dating. $\underline{I n-}$ ternational Journal of American Linguistics 21:121-137.

Thurgood, Graham. 1999. From ancient Cham to modern dialects: two thousand years of language contact and change. Oceanic Linguistics Special Publication, no. 28. Honolulu: University of Hawai'i Press.

Troike, Rudolph C. 1969. The glottochronology of six Turkic languages. International Journal of American Linguistics 35:183-191.

Tsang, Cheng-hwa. 2005. Recent discoveries at a Tapenkeng culture site in Taiwan: implications for the problem of Austronesian origins. In The peopling of East Asia: putting together archaeology, linguistics and genetics. Laurent Sagart, Roger Blench, and Alicia Sanchez-Mazas, eds. Pp. 63-73. London: RoutledgeCurzon. [RB]

Uspenskij, B. A. 2002. Istorija russkogo literaturnogo jazyka (X-XVII vv.). Moscow: Aspect. [JN]

Vajda, Edward J. Forthcoming. Yeniseic substrates and typological accommodation in Central Siberia. In Hunter-gatherers and linguistic history: a global perspective. Tom Güldemann, Patrick McConvell, and Richard Rhodes, eds. Cambridge: Cambridge University Press.

Watson, Alaric. 1999. Aurelian and the third century. London: Routledge.

Weiers, Michael. 2003. Moghol. In The Mongolic languages. Juha Janhunen, ed. Pp. 248-264. London: Routledge.

Whistler, Kenneth W. 1983. Pomo prehistory: a case for archaeological linguistics. Journal of the Steward Anthropological Society 15: 64-98.

Wichmann, Søren. 2006. Mayan historical linguistics and epigraphy: a new synthesis. Annual Review of Anthropology 35:279-294.

Wichmann, Søren, and Eric W. Holman. 2009. Population size and rates of language change. Human Biology 81:259-274.

Wichmann, Søren, Eric W. Holman, Dik Bakker, and Cecil H. Brown. 2010a. Evaluating linguistic distance measures. Physica A 389: 3632-3639.

Wichmann, Søren, André Müller, Viveka Velupillai, Cecil H. Brown, Eric W. Holman, Pamela Brown, Sebastian Sauppe, et al. $2010 b$. The ASJP database (ver. 13). http://email.eva.mpg.de/ wichmann/ languages.htm (accessed August 2010). 